\title{
Role of Systemic Inflammatory Reaction in Female Genital Organ Malignancies - State of the Art
}

\author{
Michal Mleko' \\ Kazimierz Pitynski \\ Elzbieta Pluta ${ }^{2}$ \\ Aleksandra Czerw ${ }^{3,4}$ \\ Katarzyna Sygit ${ }^{5}$ \\ Beata Karakiewicz ${ }^{6}$ \\ Tomasz Banas' \\ 'Department of Gynecology and \\ Oncology, Jagiellonian University Medical \\ College, Krakow, Poland; ${ }^{2}$ Department of \\ Radiotherapy, Maria Sklodowska-Curie \\ Institute - Oncology Centre, Krakow, \\ Poland; ${ }^{3}$ Department of Health \\ Economics and Medical Law, Medical \\ University of Warsaw, Warsaw, Poland; \\ ${ }^{4}$ Department of Economic and System \\ Analyses, National Institute of Public \\ Health - NIH, Warsaw, Poland; ${ }^{5}$ Calisia \\ University, Kalisz, Poland; \\ ${ }^{6}$ Subdepartment of Social Medicine and \\ Public Health, Department of Social \\ Medicine, Pomeranian Medical University, \\ Szczecin, Poland
}

Correspondence: Tomasz Banas Department of Gynecology and Oncology, Jagiellonian University Medical College, 2nd Jakubowskiego Street, Krakow, Poland

Tel +48 I2 4248584

$\mathrm{Fax}+48 \quad 124248560$

Email tomasz.I.banas@uj.edu.pl

\begin{abstract}
Systemic inflammatory reaction (SIR) is an unfavorable prognostic factor in many malignancies and has a role in all stages of the neoplastic process: initiation, promotion, and disease progression. Analysis of SIR can be performed by assessing indicators (eg, lymphocyte-to-neutrophil, platelet-to-lymphocyte, and monocyte-to-neutrophil ratios) and products of neutrophils and lymphocytes (ie, the systemic immune-inflammation index), or by examining the relationship between levels of $\mathrm{C}$-reactive protein and albumin (based on the Glasgow Prognostic Score, modified Glasgow Prognostic Score, and C-reactive proteinto-albumin ratio). Risk stratification is essential in the clinical management of cancer; hence, the evaluation of these factors has potential applications in the clinical management of patients with cancer and in the development of new therapeutic targets. This review summarizes the current knowledge on SIR indicators and presents their clinical utility in malignancies of the female genital organs.
\end{abstract}

Keywords: breast cancer, cervical cancer, corpus uteri cancers, C-reactive protein, monocyte-to-lymphocyte ratio, neutrophil-to-lymphocyte ratio, platelet-to-lymphocyte ratio, systemic inflammatory reaction, vulvar cancer, vaginal cancer

\section{Introduction}

The immune system has a two-fold role in the pathophysiology of neoplastic diseases. It is protective against cancer, while contributing to its progression. ${ }^{1}$ In addition, cancer cells have the ability to escape from immune surveillance and inhibit the immune response through induction of immune tolerance. ${ }^{2-4}$ Chronic inflammation may contribute to the development of many cancers. Data indicate a significant influence of systemic inflammatory response (SIR) in the formation of the neoplastic microenvironment. ${ }^{5-7}$ SIR participates in all stages of the neoplastic process development: initiation, promotion, and progression of the disease. ${ }^{8}$

The importance of inflammation in neoplastic diseases was first reported in the 19th century, when Virchow discovered the presence of leukocytes in neoplastic tissues. ${ }^{9}$ In recent years, numerous clinical studies have confirmed that SIR is an important indicator of survival in various types of cancers, including lung, ${ }^{10,11}$ colon, ${ }^{12,13}$ gastrointestinal, ${ }^{14,15}$ liver, ${ }^{16,17}$ esophageal, ${ }^{18,19}$ breast, ${ }^{20-22}$ ovarian, ${ }^{23-25}$ cervical, ${ }^{26,27}$ pancreatic, ${ }^{28}$ prostate, ${ }^{29}$ kidney, ${ }^{30}$ and bladder cancers. ${ }^{31}$ It must be emphasized, however, that increased systemic inflammation is not pathognomonic solely for malignancies. Increased SIR is predominately observed in autoimmune diseases including rheumatoid arthritis, ${ }^{32-34}$ spondyloarthropathies, ${ }^{35,36}$ Crohn's disease, ${ }^{37}$ diabetes, ${ }^{38}$ and Hashimoto's thyroiditis ${ }^{39}$ and has even been reported in 
patients with COVID-19. ${ }^{40}$ Furthermore, multiple demographic and lifestyle factors, including sex, age, race, marital status, body mass index, physical activity, smoking history, and alcohol consumption are independently associated with inflammatory biomarkers; these should be also taken in consideration to more accurately assess their prognostic power. ${ }^{41}$

In the first part of this review, we aim to describe the role of tumor microenvironment markers used for SIR evaluation. In the second part, we discuss recent advances in gynecological and breast cancers in relation to wellestablished cancer markers. In addition we review the potential predictive value of markers as independent risk factors for disease-free survival (DFS) and overall survival (OS) and their possible use in everyday clinical practice.

\section{Strategy and Selection Criteria}

Four indexed databases, PubMed, Embase, Scopus, and Web of Science, were searched from January 1, 2000 to December 31, 2020 using the keywords "breast cancer," "ovarian cancer," "endometrial cancer," "cervical cancer," "vaginal cancer," and "vulvar cancer," as well as "systemic inflammatory reaction," "systemic inflammatory response," "neutrophil-to-lymphocyte ratio," "monocyteto lymphocyte ratio," "platelet-to-lymphocyte ratio," "C-reactive protein-to-albumin ratio," "fibrinogen-to albumin ratio," "neutrophils and lymphocytes-to-platelets," "systemic inflammatory index," "Glasgow Prognostic Score," and "Modified Glasgow Prognostic Score." Medical subject-heading terms used included "colonic neoplasms" and "neoadjuvant therapy." Relevant papers were included if they had been published in English or Polish and specifically addressed the aim of the study. Studies were excluded if they were conference abstracts or not published in peer review journals. This paper is constructed as a narrative review rather than a systematic review. In our opinion this allows full discussion of important background details.

\section{Pathophysiology of the Tumoral Inflammatory Microenvironment and Systemic Inflammatory Reaction in Neoplasms}

There is a significant relationship between chronic inflammation and predisposition to tumor development in many types of cancer. ${ }^{5}$ Neoplastic cells secrete various cytokines and chemokines, including interleukin 1 (IL1), IL6, tumor necrosis factor-alpha (TNFA), and IL23, which recruit inflammatory cells (eg, granulocytes, macrophages, and mast cells). ${ }^{6,7}$ Pro-inflammatory cytokines are involved in the formation and development of neoplasms. They also adversely affect patients with cancer by increasing fatigue and pain, inducing toxicity and resistance to treatment, and enhancing the development of cachexia and anorexia. ${ }^{23,42}$

Macrophages have a dominant role in the inflammatory response. ${ }^{43-45}$ Macrophages infiltrate malignant tumor tissues in high numbers (so-called [TAMs]) and may account for $>50 \%$ of the tumor mass. ${ }^{46}$ Macrophages undergo a characteristic phenotypic transformation under the influence of cytokines, predominately $1 \mathrm{~L} 10$ and TNFB secreted by cancer cells. ${ }^{47}$ Macrophages are transformed from phagocytes that present antigens and produce pro-inflammatory cytokines (a typical phenotype of M1 cells) into cells that secrete anti-inflammatory and immunosuppressive cytokines (M2 phenotype). Studies using human tumor samples have demonstrated that a higher density of macrophages, especially macrophages with the M2 phenotype, is closely associated with worse clinical prognosis in many malignant tumor types. ${ }^{48}$ TAMs are phenotypically and functionally differentiated cells. ${ }^{49,50}$ Most TAMs in the tumor microenvironment are closely related to the M2-like phenotype. Depending on their location, TAMs produce various types of mediators, ${ }^{50}$ including proangiogenic factors such as vascular endothelial growth factor (VEGF), TNFA, and IL8; others secrete different types of growth factors and other proteins involved in proteolytic modification of the extracellular matrix. TAMs also participate in the migration of neoplastic cells through the secretion of chemotactic factors. ${ }^{49}$ TAMs can stimulate tumor cell proliferation, migration, and genetic instability. Acting at the primary tumor site or at sites of secondary localization, they promote invasion and metastasis. TAMs are generally associated with poor prognosis in most human cancers, ${ }^{51}$ this is in stark contrast with the traditional notion that macrophages have host-protecting roles in inflammatory microenvironments. When exposed to signals from the tumor microenvironment, macrophages show a surprising degree of plasticity in functional reprogramming and adopt either pro- or anti-inflammatory phenotypes in response to environmental stimuli. ${ }^{51}$ Available information suggests that TAMs infiltrating established tumors acquire the properties of an M2-like phagocytic population, including promotion of tumor growth and angiogenesis, remodeling of tissues, and suppression of anti-tumor immunity. ${ }^{52}$ Inflammation in the neoplastic microenvironment also causes vascular 
changes; there is increased permeability of existing vessels and formation of new vessels due to, for example, VEGF. ${ }^{6}$ TAMs promote angiogenesis and lymphoangiogenesis, as well as tissue remodeling with fibrous tissue deposition.

Pro-inflammatory cytokines secreted by cancer cells also increase levels of reactive oxygen species (ROS) and their derivatives, which cause DNA damage and, in excessive amounts, intensify the process of neoplastic transformation. ${ }^{6}$ Oxidative stress arising in neoplastic tumors as a result of a disturbance of the pro- and antioxidant balance initiates inflammation, which leads to the further development of neoplastic disease. ${ }^{53}$ Secondary production of ROS is induced by inflammation through feedback. ${ }^{54}$ The role of ROS in cancer cells depends on their levels. Small amounts of ROS act as signaling molecules, inducing carcinogenesis and heterogeneity. In large amounts, they can act as tumor modulators, exerting a genotoxic or even pro-apoptotic effect on neoplastic cells. $^{55}$

In addition to macrophages, neutrophils in the tumor environment can undergo phenotypic transformation (ie, they become tumor-associated neutrophils [TANs]), depending on the signals received from neoplastic cells. Thus, as well as their anti-tumor activity, they may have pro-tumor activity and can thus initiate tumor development, progression, or metastasis. ${ }^{56}$ TANs are very similar to TAMs, and the two together constitute the essential components of the tumor microenvironment. ${ }^{57}$

Experimental studies of the neoplastic microenvironment have demonstrated the presence of two subpopulations of neutrophils in the circulation: 1) mature, low-volume, and high-density cells (ie, high-density neutrophils), exhibiting anti-tumor activity (N1 phenotype); and 2) a largevolume and low-density heterogeneous population with the N2 phenotype (ie, low-density neutrophils), as well as immature bone marrow-derived neutrophils such as myeloid suppressor cells (ie, granulocytic myeloid-derived suppressor cells). Under physiological conditions, high-density neutrophils account for approximately $95 \%$ of the population. In the tumor environment, the percentage of low-density neutrophils rapidly increases, potentially rendering them the dominant fraction in the circulation. ${ }^{58,59}$

\section{Inflammatory Markers}

The severity of inflammation can be assessed based on a variety of biochemical markers. Potential hematological biomarkers representing SIR in patients with cancer include $\mathrm{C}$-reactive protein (CRP), albumins, and changes in blood counts. As hematology tests are routinely performed in the majority of patients with cancer, these biomarkers can be used as easily measurable and inexpensive parameters to determine SIR severity in patients with cancer.

CRP is synthesized by hepatocytes. It is a non-specific yet sensitive marker of acute inflammatory response and is expressed in selected neoplastic cells. ${ }^{60}$ Numerous studies have indicated that an increased CRP level value is an indicator of poor prognosis in various types of cancer. $^{11,16,18,24,29,30}$ The correlations between the expression of this marker and the risk of cancer progression and long-term survival are not fully understood. Several proinflammatory cytokines (eg, IL1, IL6, and TNFA) produced by neoplastic cells induce CRP synthesis by the liver and other tissues. ${ }^{8,9}$ CRP may accelerate angiogenesis by increasing levels of vascular growth factors and ILs, thereby increasing tumor cell invasion and metastasis by binding to integrins in the neoplastic microenvironment. ${ }^{61,62}$ Increased levels of CRP in serum, detected prior to the initiation of therapy, may indicate a high degree of tumor aggressiveness and may be associated with resistance to treatment and poor treatment outcomes in patients. $^{63}$

Albumin, another marker of acute inflammatory response, is generally used for assessing nutritional status. ${ }^{60}$ Malnutrition and inflammation suppress albumin synthesis, thereby reducing immune defense, impeding treatment response, and contributing to adverse outcomes in patients with cancer. ${ }^{64}$ Low serum albumin concentrations may be due to the production of cytokines (eg, IL6), which modulate albumin production by hepatocytes. ${ }^{60}$ Alternatively, TNFA can increase the permeability of the microcirculation, thereby enhancing the transcapillary passage of albumins. Moreover, the presence of micrometastases of neoplastic cells in the liver may induce the production of various cytokines (IL1, IL6, and TNFA) by Kupffer cells that can modulate albumin synthesis by hepatocytes. $^{8,9}$ Hypoalbuminemia is rare in the early stages of cancer; however, as the disease progresses, levels of albumin are significantly decreased and serve as prognostic indicators in patients with various types of cancer. $^{13,20,23}$ Low serum albumin levels prior to the initiation of anti-cancer therapy represent an independent indicator of poor prognosis in patients with cancer. ${ }^{65}$ Improvement in nutritional status has positive effects on quality of life, response to cancer therapy, and survival of patients with cancer. $^{66}$ 
Changes in white blood cell levels also occur during SIR. Neutrophilia, thrombocytosis, and lymphocytopenia can be observed in the peripheral blood of patients with cancer. ${ }^{56,67-69}$ Numerous studies have shown that cancerassociated leukocytosis and/or neutrophilia and lymphocytopenia are unfavorable prognostic factors in these patients. $^{70,71}$

As well as changes in white blood cell parameters, thrombocythemia is also found in patients with cancer, and its degree depends on the advancement of the neoplastic process. ${ }^{72,73}$ Thrombocytosis increases the risk of metastases and is also an indicator of poor prognosis. It is thought that the increase in the number of platelets may be the result of increased secretion of a number of cytokines by the tumor, which may induce thrombopoiesis. ${ }^{73}$

In addition to the absolute values of blood counts, the ratios of particular types of white blood cells, such as the lymphocyte-to-neutrophil ratio (NLR), platelet-tolymphocyte ratio (PLR), and lymphocyte-to-monocyte ratio (LMR), as well as the systemic immuneinflammation index (SII) and CRP-to-albumin ratio (CAR), are important in the assessment of prognosis in cancers.

NLR has attracted considerable interest over the past decade as a potential prognostic factor in various types of cancer and as an indicator for risk stratification of patients with cancer. ${ }^{22,25,27}$ Pretreatment NLR values were elevated in patients with neoplasms of various organ localizations (eg, colorectal, lung, ovarian, and esophageal). ${ }^{25,74-76}$ In many studies, pretreatment NLR values have been considered to be prognostic factors related to OS and asymptomatic survival. ${ }^{77}$ Higher NLR values are observed in more advanced stages of the neoplastic process, where SIR is markedly intensified. ${ }^{77}$

Similar to NLR, LMR and PLR may reflect abnormalities in the immune system and, thus, abnormal anti-tumor activity. ${ }^{8}$ Lower LMR has been associated with poor survival in both non-hematological and hematological malignancies. $^{78}$

The ratio of neutrophils and lymphocytes to platelets (ie, SII) is also used to assess prognosis in various types of cancer. Increased SII is associated with a worse prognosis in patients with colorectal, liver, pancreatic, and bladder cancers; it is also linked to a higher frequency of disease recurrence. ${ }^{79}$ Elevated SII reflects changes in the cancer microenvironment that favor disease progression and spread. Moreover, SII values are correlated with poor histological differentiation of the tumor, larger tumor size, and more advanced stage of TNM. ${ }^{79}$ SII was first described by $\mathrm{Hu}$ et al in hepatocellular carcinoma. Increased SII values were shown to be associated with worse prognosis, which the authors of the study attributed to several factors. Research has shown that patients with an elevated SII value usually have thrombocytosis, neutropenia, or lymphopenia prior to undergoing surgery. ${ }^{80}$ Neutrophils secrete various inflammatory mediators that influence the promotion and proliferation of neoplastic cells, thereby increasing invasion and metastasis to lymph nodes or distant organs. ${ }^{80}$ Platelets secrete chemotactic factors for cancer cells, induce the creation of optimized conditions for metastases, cause epithelial transformation, and increase the number of circulating tumor cells. ${ }^{80}$ Lymphopenia contributes to the avoidance of immune surveillance by cancer cells and reduces the damage caused by the autoimmune response. ${ }^{80}$ Collectively, these factors explain the role of SII in promoting the progression of neoplastic disease and its association with worse prognosis.

CAR was initially assessed in patients with acute diseases and sepsis. ${ }^{81,82}$ Recently, its prognostic value has gained importance in cancers including hepatocellular carcinoma, ${ }^{83}$ gastric cancer, ${ }^{84}$ and esophageal squamous cell carcinoma, ${ }^{85}$ as well as ovarian, ${ }^{86}$ breast, ${ }^{87}$ and cervical cancers. ${ }^{87}$ Elevated preoperative CAR levels are thought to be associated with poor survival of patients with these tumor types. Moreover, the CAR index may serve as a prognostic biomarker of cardiotoxicity during anti-cancer therapy. ${ }^{88}$

Other useful markers of inflammation include the Glasgow Prognostic Score (GPS) and Modified GPS (mGPS), which are calculated based on the concentrations of CRP and albumin.

GPS was first described in 2003 by Forrest et al, who demonstrated its prognostic value in non-small-cell lung cancer. ${ }^{89}$ GPS is defined by hypoalbuminemia $(<35 \mathrm{~g} / \mathrm{L})$ and elevated levels of CRP ( $>10 \mathrm{mg} / \mathrm{L})$ : if both parameters are abnormal, the assigned value is 2 ; if one of them is abnormal, the assigned value is 1 ; and if both parameters are normal, the assigned value is $0 .{ }^{89}$

Some researchers use mGPS to evaluate prognostic outcomes in cancer. This score is also calculated using levels of CRP and albumin. Patients with CRP $<10 \mathrm{mg} / \mathrm{L}$ receive 0 points; those with $\mathrm{CRP}>10 \mathrm{mg} / \mathrm{L}$ are assigned 1 point; and those with $\mathrm{CRP}>10 \mathrm{mg} / \mathrm{L}$ and albumin $<35 \mathrm{~g} / \mathrm{L}$ receive 2 points. ${ }^{89}$ 
Numerous studies have shown a relationship between increased GPS/mGPS and shorter OS in various types of cancer (eg, colon, esophageal, liver, stomach, and pancreatic). ${ }^{90}$ GPS and mGPS are particularly important prognostic indicators for use before initiation of anticancer therapy. Patients with low GPS and mGPS prior to treatment were shown to have better prognosis versus those with elevated scores. ${ }^{89}$ Hence, the GPS/mGPS indicators represent cost-effective and available markers for risk stratification in neoplastic disease, particularly before the initiation of anti-neoplastic treatment. Thus, they may facilitate clinical decision-making and improve outcomes in patients with tumors.

The fibrinogen-to-albumin ratio (FAR) and fibrinogen and NLR (F-NLR) are new inflammatory markers that may have important roles in the assessment of prognosis in patients with cancer. Fibrinogen and albumin play important parts in the inflammatory response. Fibrinogen, a glycoprotein synthesized by hepatocytes, is produced in response to inflammatory cytokines. Similar to CRP, fibrinogen (a protein involved in the acute inflammatory response) plays an important part in blood clotting. Therefore, it is involved in maintaining normal hemostasis in the body. Both the SIRand the hemostatic system are closely related to the development of neoplastic diseases. ${ }^{8,90}$ Fibrinogen has the ability to directly bind to VEGF, transforming growth factor $\beta$, platelet-derived growth factor, and fibroblast growth factor. Therefore, it has important roles in cell proliferation, epithelial transformation, angiogenesis, and tumor cell metastasis. ${ }^{91,92}$ Research has shown that elevated plasma fibrinogen levels indicate poor prognosis in various types of cancer, including esophageal squamous cell carcinoma and small-cell liver carcinoma. ${ }^{93}$ Perisanidis et al proposed a treatment that lowers the concentration of fibrinogen in plasma for prolonging survival in patients with solid tumors. ${ }^{94}$ Moreover, it has been suggested that therapies targeting fibrinogen-dependent interactions may positively contribute to the treatment of some types of malignant neoplasms. ${ }^{95}$

The role of albumin in the inflammatory response has been previously described in this paper. As already mentioned, the prognostic value of the albumin level before the initiation of anti-tumor treatment has been reported in various malignant neoplasms (eg, kidney, lung, ovarian, and stomach). ${ }^{93}$ It has also been demonstrated that hypoalbuminemia is associated with poor treatment outcomes in patients with cancer. ${ }^{93}$
Recent studies have shown that FAR is a potential new prognostic tool for various cancers, including esophageal squamous cell carcinoma, ${ }^{96}$ as well as being an independent prognostic factor for hepatocellular carcinoma following surgery. ${ }^{93}$ Elevated FAR may also indicate an increased risk of recurrence of liver tumors. ${ }^{93}$

F-NLR is another new predictive marker for cancer. As previously mentioned, neutrophils promote tumor development, invasion, and metastasis by secreting inflammatory mediators and external factors, thereby altering the neoplastic microenvironment. Lymphocytes inhibit tumor proliferation and metastasis, induce cytotoxic cell death, and produce inhibitory cytokines. Thus, lymphopenia may cause an insufficient immune response to malignant neoplasms, which facilitates tumor progression and is consequently associated with a poor prognosis. ${ }^{97}$ Elevated fibrinogen inhibits the elimination of cancer cells by cytotoxic cells or natural killer cells. Thus, the F-NLR index reflects changes in the neoplastic microenvironment and the inflammatory response in tumors. Recent studies have indicated that elevated F-NLR levels are associated with worse prognosis and clinical outcomes in patients with malignant neoplasms, including lung, stomach, esophageal, and colorectal cancers. ${ }^{97}$ In addition, in colorectal cancer, F-NLR was associated with a more advanced T stage of the tumor, larger tumor size, and more invasion. Thus, elevated F-NLR may promote tumor proliferation, invasion, and metastasis. ${ }^{97}$

\section{Systemic Inflammatory Reaction in Neoplasms of the Female Genital Organs}

\section{Breast Cancer}

In developed countries, breast cancer is the most common malignancy in females, with a peak incidence observed after 40 years of age. ${ }^{98}$ Based on a meta-analysis of 15 studies that included 8563 women with breast cancer, Ethier et al confirmed that high NLR (cutoff point $>2.5$ or 3.0 depending on the analyzed groups) was associated with worse OS (hazard ratio [HR]: 2.56, 95\% confidence interval $[\mathrm{CI}]: 1.96-3.35 ; \mathrm{p}<0.001)$ and DFS (HR: 1.74 , 95\% CI: $1.47-2.07 ; \mathrm{p}<0.001) .{ }^{99}$ In addition, higher prognostic value for DFS was observed in patients with triplenegative breast cancer versus those with other subtypes. ${ }^{99}$ This correlation was confirmed in studies including only patients with early-stage disease, as well as in those comprising patients with both early-stage and advanced 
disease. Similarly, Koh et al analyzed 157 women with hormone receptor-positive but human epidermal growth factor receptor 2 (HER2)-negative breast cancer. They confirmed that NLR was an independent prognostic factor for OS (HR: 24.87; 95\% CI: 3.1-201.3; $\mathrm{p}=0.003$ ). ${ }^{100}$ However, a recent review by Corbeau et al showed limited predictive value of NLR for OS and pathological complete response in both patients with early-stage breast cancer receiving neo-adjuvant chemotherapy and those with advanced breast cancer. ${ }^{101}$ On the contrary, Cullinane et al concluded that a lower NLR was associated with a higher rate of pathological complete response (odds ratio [OR]: $1.83,95 \% \mathrm{CI}: 1.15-2.91 ; \mathrm{p}=0.0003$ ) and a higher 5-year DFS; however, the difference did not reach statistical significance. ${ }^{102}$ The association between NLR and OS has been confirmed in many studies. Nevertheless, it must be emphasized that this association was mostly noted in univariate analyses, whereas multivariate analyses often failed to show that NLR was an independent predictor of OS. ${ }^{103-105}$ These results should be interpreted with caution because a potential bias caused by heterogeneity in the study groups may have significantly influenced the multivariate analyses.

Among other inflammatory blood markers, PLR and LMR were investigated in females with breast cancer. Cho et al analyzed NLR, derived NLR (dNLR), PLR, and LMR in the immediate preoperative period in female patients treated for breast cancer with breast-conserving surgery. ${ }^{106}$ They analyzed the relationship between the aforementioned inflammatory markers and clinicopathologic variables, as well as disease-specific survival (DSS) and DFS. Based on their analyses, they developed a nomogram to predict 3- and 5-year DSS in patients with breast cancer. In the univariate analysis, high NLR, dNLR, PLR, and low LMR were significantly associated with poor DSS and DFS. In the multivariate analysis, only PLR (HR: 3.226, 95\% CI: $1.768-5.885$ and HR 1.824, 95\% CI: 1.824-6.321 for DSS and DFS, respectively) was still identified as an independent predictor of outcomes. ${ }^{106}$ Araki et al and Vernieri et al confirmed through univariate analysis that high levels of PLR and LMR are negative predictors of progression-free survival (PFS) in breast cancer survivors. ${ }^{107-109}$ Additionally, Ji and Wang analyzed nine studies involving 2724 patients, revealing that increased SII with a cutoff point of 600 increased the risk of poorer OS (HR: 1.98, CI: 1.31-2.99; $\mathrm{p}=0.001$ ) and DFS (HR: 2.05, CI: $1.30-3.24 ; \mathrm{p}=0.002)$, as well as lymph node involvement (HR: 1.35 , CI: $1.15-1.59 ; \mathrm{p}<0.001$ ) in female patients with breast cancer, particularly in the triplenegative subgroup. ${ }^{109}$ Chen et al reported that SII was an effective prognostic indicator of OS and DFS in female patients with breast cancer undergoing neo-adjuvant chemotherapy. ${ }^{110}$ By analyzing 262 patients with breast cancer, they defined the optimal cutoff value of SII using a receiver operating characteristic curve at a level of $0.85 \times 10^{9} /$ L. Patients with low SII had longer DFS and OS (41.27 vs 30.45 months, respectively, HR: 1.694, 95\% CI: $1.128-2.543, \mathrm{p}=0.011 ; 52.86$ vs 45.75 months, respectively, HR: $1.288,95 \% \mathrm{CI}: 0.781-3.124, \mathrm{p}=0.002$ ), as well as better 3-, 5-, and 10-year DFS and OS rates. ${ }^{110}$

Most studies examining the prognostic value of SIR in patients with primary breast cancer have shown that NLR, PLR, and LMR are significantly associated with DSS and DFS. PLR accurately predicted individualized survival probability in breast cancer; thus, this practical model could support clinicians and patients in clinical decisionmaking and treatment optimization. ${ }^{102}$

In patients undergoing potentially curative surgery for primary operable breast cancer, preoperative concentrations of albumin predict survival, independent of tumor-based factors. ${ }^{104}$ Moreover, GPS was significantly associated with breast cancer-specific survival and appeared to be a useful indicator of poor outcome independent of treatment in patients with metastatic cancer.

There is growing evidence that TAMs are associated with poor prognosis in breast cancer. Over the years, studies of the role of TAMs in breast cancer progression have found them to be capable of inducing angiogenesis, remodeling the tumor extracellular matrix to aid invasion, modeling breast cancer cells to evade the host immune system, and recruiting immunosuppressive leukocytes to the tumor microenvironment. ${ }^{111}$ As well as these functions, a potential role for TAMs in the activation of breast cancer stem cells has emerged. Thus, TAMs in breast cancer can enhance cancer cell invasion by degrading the extracellular matrix; they can also stimulate tumor vascularization and angiogenesis, and suppress the anti-tumor functions of cytotoxic $\mathrm{T}$ cells, resulting in poor prognosis. ${ }^{111} \mathrm{~A}$ meta-analysis of 16 studies showed that high density of TAMs was related to worse OS and DFS. ${ }^{111} \mathrm{CD} 68+$ which is a cell surface marker of TAMs, was associated with breast cancer characteristics indicative of poor prognosis: larger tumor size, higher tumor grade, lymph node metastasis, vascular invasion, hormone receptor negativity, HER2 expression, and basal phenotype. ${ }^{111}$ Another marker of TAMs, CD163, was also an 
independent prognostic factor for worse DFS and/or OS in patients with breast cancers. ${ }^{111}$ In summary, TAMs have a significant role in breast cancer. Targeted therapies directly targeting TAMs could therefore improve survival rates of breast cancer patients.

\section{Endometrial Cancer}

Endometrial cancer is the most common type of uterine corpus malignancy. This type of cancer has a high incidence rate in developed countries; it shows a trend of increasing incidence but is fortunately associated with a low mortality rate. ${ }^{112}$ Endometrial intraepithelial neoplasia, previously defined as endometrial hyperplasia with atypia, is an established precursor of endometrioid endometrial cancer. A significantly elevated mean NLR was observed by Cakmak et al in females with endometrial hyperplasia with atypia, in comparison with patients with endometrial hyperplasia without atypia and controls $(6.5$ [0.6-26.6] vs $3.3 \quad[0.6-26.6]$ vs 2.4 [0.6-14.0]; $\mathrm{p}=0.004) .{ }^{112}$ Similarly, they observed significantly increased mean PLR values in patients with endometrial hyperplasia with atypia versus those with simple endometrial hyperplasia and controls (182.9 [75.4-459.0] vs 159.9 [47.1-516.6] vs 126.8 [41.4-312.8], respectively; $\mathrm{p}=0.024) .{ }^{113}$ Ural et al observed elevated NLR only in patients with endometrial cancer versus those with endometrial hyperplasia and healthy controls $(3.8 \pm 3.7$ vs 3.3 \pm 4.97 vs $2.5 \pm 2.52$, respectively; $\mathrm{p}=0.024) .{ }^{114}$ Furthermore, they did not observe significant changes in PLR between the three analyzed groups. ${ }^{114}$ Subsequently, Selen et al confirmed in a multivariate analysis that low PLR $(\leq 133.3)$ was related to a statistically significant OR $(8.01 ; \mathrm{p}<0.001)$ for the diagnostic prediction of lowgrade endometrioid endometrial cancer compared with endometrial intraepithelial neoplasia. ${ }^{115}$ Acmaz et al investigated the diagnostic utility of elevated NLR and PLR in females experiencing abnormal bleeding. ${ }^{116}$ Although the NLR was significantly higher in patients with cancer than in those with endometrial atypia and controls ( $p=0.001$ and $p=0.025$, respectively), there was no difference between patients with simple endometrial hyperplasia and controls. ${ }^{116}$ The PLR was significantly lower in controls than in the other groups $(p<0.001)$; however, there was no significant difference between patients with hyperplasia and those with cancer. ${ }^{116}$ A meta-analysis performed by Pergialiotis et al confirmed that females with endometrial carcinoma had significantly higher NLR compared with healthy controls. ${ }^{117}$ The first therapeutic option for females suffering from endometrial cancer is surgery. ${ }^{118}$ Pretreatment NLR was shown to be an accurate predictor of DFS and OS in endometrial cancer survivors. ${ }^{119}$ In a univariate analysis, Haruma et al showed that NLR $>2.412$ is a predictor of poor DFS and OS (HR: 2.365, 95\% CI: 1.341-4.173; $\mathrm{p}=0.003$ and HR: 4.088, 95\% CI: 1.945-8.590; $\mathrm{p}<0.001$ ), whereas PLR (cutoff value: 175.72) was only significant for the prediction of OS (HR: 2.054, 95\% CI: 1.021-4.132; p=0.043). ${ }^{119}$ Kadan et al found that the mean NLR was higher in the node-positive group compared with node-negative patients (3.4 vs 2.9, respectively); however the difference was not statistically significant. ${ }^{120}$ Cong et al revealed that NLR and PLR, as well as LMR, are valuable predictors of OS in patients with endometrial cancer. They demonstrated in a multivariate analysis that high NLR $>2.14$ (HR: 2.71, 95\% CI: 1.83-4.02; p<0.001), high PLR >131.82 (HR: 2.75, 95\% CI: 1.90-3.97; $<<0.001)$, and high $\mathrm{LMR}>0.22$ (HR: 1.72, 95\% CI: 1.20-2.45; p=0.003) were significantly associated with worse OS. The combined indicator, high NLR + high PLR + high LMR (HR: 4.34, 95\% CI: $2.54-$ $7.42 ; \mathrm{p}<0.001)$, showed the highest prognostic value. ${ }^{121}$ Eo et al analyzed 255 females with newly diagnosed endometrial cancer and reported an LMR cutoff value of 3.28 for both DFS and OS. ${ }^{122}$ The 5-year DFS rates in the LMR-low and LMR-high groups were $64.5 \%$ and $93.9 \%$, respectively $(\mathrm{p}<0.0001)$. The 5 -year OS rates in the two groups were $76.7 \%$ and $96.5 \%$, respectively $(p<0.0001)$. Thus, LMR is a strong prognostic factor predicting DFS and OS ( $p<0.0001$ and $p<0.0001$, respectively) in endometrial cancer survivors. ${ }^{122}$

In addition, using a Cox regression model, $\mathrm{Li}$ et al identified CRP and D-dimer as independent prognostic indicators of poor OS. The HRs for CRP and D-dimer were 0.215 (95\% CI: $0.084-0.549)$ and 0.252 (95\% CI: $0.095-0.670)$, respectively. ${ }^{123}$

Substantial amounts of TAMs and regulatory $\mathrm{T}$ cells were also detected in endometrial cancer. The increased infiltration of TAMs was proportionally associated with advanced International Federation of Gynecology and Obstetrics (FIGO) stage, high tumor grade, increased lymph vessel density, lymphovascular space invasion, and lymph node metastasis. ${ }^{124}$ Thus, the presence of TAMs indicated aggressive tumor behavior and appeared to be an independent prognostic factor for recurrence-free survival. ${ }^{124}$ In addition, type 2 endometrial cancers were found to have nearly twice the TAM density of type 1 cancers. The CD68+ stromal TAM density was higher in 
patients with type 2 cancers compared with those with type 1 cancers. This difference may be due to the predominance of M1 macrophages in the stroma of type 2 cancers. $^{125}$

\section{Ovarian Cancer}

The female reproductive organs provide favorable conditions for the development of chronic inflammation. Ovulation is associated with cyclic damage and restoration of the epithelium covering the ovary, as well as frequent inflammation of the pelvic organs, endometriosis, or polycystic ovary syndrome, which generate conditions favorable for the activation of a number of pro-inflammatory mediators. ${ }^{98,99,126}$ Research suggests that ovarian cancer is usually accompanied by systemic immunosuppression.

NLR, LMR, and PLR may be helpful indicators for differentiating benign neoplasms from malignant changes in the ovaries. Moreover, they are sensitive indicators correlated with local advancement and response to firstline chemotherapy. High NLR and PLR, as well as low LMR prior to treatment initiation, are associated with a significantly higher risk of disease progression in patients with ovarian cancer. ${ }^{100}$ In a retrospective metaanalysis of 13 studies involving 3467 patients with ovarian cancer, Zhao et al found that high NLR was associated with poor OS and PFS (pooled HR: $1.70,95 \%$ CI: $1.35-$ 2.15; and HR: $1.77,95 \%$ CI: $1.48-2.12$, respectively). ${ }^{127}$ They also demonstrated that high PLR adversely affected OS and PFS in ovarian cancer (pooled HR: $2.05,95 \% \mathrm{CI}$ : 1.70-2.48; and HR: 1.85 , 95\% CI: $1.53-2.25$, respectively). ${ }^{127}$ Similarly, Huang et al conducted a systematic review and meta-analysis of 12 observational studies involving 3854 patients with ovarian cancer. They showed that a high pretreatment NLR was significantly associated with shorter OS (HR: 1.69, 95\% CI: 1.29-2.22) and PFS (HR 1.63, 95\% CI: 1.27-2.09). ${ }^{128}$ Elevated NLR was also significantly correlated with advanced FIGO stage (OR: 2.32, 95\% CI: 1.79-3.00), increased serum cancer antigen 125 (CA-125) concentration (OR: 3.33, 95\% CI: 2.43-4.58), more extensive ascites (OR: 3.54, 95\% CI: $2.31-5.42)$, and poorer response to chemotherapy (OR: 0.53 , 95\% CI: $0.40-0.70) .{ }^{129}$ Increased NLR was also confirmed in ovarian cancer patients with low CA125 levels $(<35 \mathrm{U} / \mathrm{mL}) .{ }^{129}$ In this challenging group of patients, NLR $>1.72$ showed increased sensitivity, with appropriate specificity and a higher negative predictive value. ${ }^{129}$ The dNLR is defined as a number of neutrophils divided by the difference between lymphocytes and neutrophils. In a study of 262 patients with ovarian cancer, 258 patients with benign ovarian disease, and 232 healthy controls, Wu et al reported that the highest median NLR was observed in patients with ovarian cancer compared with the other two groups $(2.29$ [1.50-3.55] vs 1.44 [1.141.88] vs 1.29 [1.06-1.54], respectively; $\mathrm{p}<0.001) .{ }^{130}$ Furthermore, at a cutoff value of $\leq 2.11$, dNLR was able to distinguish ovarian cancer from benign ovarian disease. At a cutoff value of $\leq 1.9$, dNLR showed diagnostic power for distinguishing patients with ovarian cancer from healthy controls. ${ }^{130}$ Moreover the dNLR value increased in parallel with the stage of ovarian cancer and was positively correlated with serum concentration of CA-125 $(\mathrm{r}=0.507, \mathrm{p}<0.001){ }^{130}$

Moreover, increased values of the CAR have been observed in patients with ovarian cancer. Elevated baseline CAR is considered an independent unfavorable prognostic indicator of OS in patients with ovarian cancer. ${ }^{131}$

TAMs have been strongly implicated in the progression and chemoresistance of ovarian cancer. In ovarian cancer, the overall and intratumoral M1/M2 ratio is a relatively efficient TAM parameter for predicting the prognosis of patients, especially in those with serous-tissue-type cancer. ${ }^{132}$ By performing immunohistochemical analysis of both M1 and M2 macrophages in ovarian cancer, Zhang et al found that patients with high proportions of M1 macrophages had better prognosis, suggesting that M2 TAMs have an important role in the progression of ovarian cancer. ${ }^{132}$ TAMs exhibit immunological checkpoint modulators, such as the B7 family and programmed deathligand 1 (PD-L1), which also play an important part in the development, metastasis, and invasion of ovarian cancer; however, the underlying mechanism is poorly understood. ${ }^{133}$

\section{Cervical Cancer}

SIR also has an important role in cervical cancer, which is one of the leading causes of mortality among females worldwide. ${ }^{134}$ The use of hematologic parameters in determining prognosis and managing therapy for cervical cancer has become more common among clinicians. This approach justifies the use of NLR for estimating the mortality and recurrence rates of cervical cancer. Elevated NLR was found in patients with cervical cancer compared with healthy controls and females with precancerous cervical changes, including low- and high-grade intraepithelial neoplasia. ${ }^{135}$ Furthermore, PLR values were significantly higher in cervical cancer survivors versus those with low- and high-grade 
intraepithelial neoplasia and controls. Moreover, logistic regression analysis revealed that age (OR: 1.075, 95\% CI: 1.020-1.132; $\mathrm{p}=0.007$ ), NLR (OR: 1.643, 95\% CI: 1.009 3.142; $\mathrm{p}=0.047$ ), and PLR (OR: 1.032, 95\% CI: 1.003 $1.062 ; p=0.029)$ were predictors of the presence of cervical cancer. $^{135}$ The median NLR and PLR were significantly higher in patients with advanced-stage disease versus those with early-stage disease (7.58 [1.36-33.20] and 247.89 [97.10-707.11], respectively; $\mathrm{p}=0.001)$. Furthermore, a strong positive correlation was found between the staging of cervical cancer and the NLR value $(r=0.638)$ and PLR $(\mathrm{r}=0.668)$. Thus, increased pretreatment NLR and PLR values may provide useful information for predicting the stage of cervical cancer. ${ }^{136}$ By analyzing 1066 patients with cervical cancer, Lee et al demonstrated that pretreatment NLR $>1.9$ was correlated with poor OS. ${ }^{27} \mathrm{Zhu}$ et al confirmed that elevated NLR was linked to age, parametrial involvement, tumor invasion depth, and histologic grade of cervical cancer; and that PLR was related to age, parametrial involvement, tumor invasion depth, and FIGO stage. ${ }^{137}$ Univariate analysis identified high PLR as a significant poor predictor of PFS and OS, whereas NLR did not exhibit predictive power. $^{137}$ PLR is also used as a predictor of lymph node metastasis in cervical cancer and improves risk stratification for predicting patient survival. ${ }^{27}$

Treatment with radical surgery is used in the early stages of cervical cancer, whereas advanced cases are treated with chemoradiotherapy or radiotherapy. Zhu et al were the first to show that an increased post-/pre-radiotherapy eosinophil ratio was independently associated with worse PFS and OS in cervical cancer survivors. ${ }^{138}$ Trinh et al retrospectively reviewed patients with cervical cancer who received definitive chemoradiation therapy and found that increased LMR was negatively correlated with PFS and OS. ${ }^{139}$

Zhang et al were the first to evaluate the prognostic significance of pretreatment CAR in patients with stage IB-IIA cervical cancer undergoing radical surgery. ${ }^{140}$ At a cutoff value of 0.15 , patients with a high CAR had significantly shorter PFS and OS $(\mathrm{p}<0.001) .{ }^{140}$ Higher CAR was also significantly associated with elevated NLR and PLR $\left(\mathrm{p}<0.001\right.$ and $\mathrm{p}<0.001$, respectively). ${ }^{140}$

Other studies showed that high SII was also associated with poor prognosis in patients with cervical cancer. Higher SII had a significant correlation with larger tumors but was not correlated with other clinicopathological parameters. SII can independently predict the OS of patients with cervical cancer undergoing radical resection; thus, it is superior to the existing systemic inflammatory indices. Therefore, SII may be a reliable index for predicting the postoperative survival of patients with cervical cancer. ${ }^{101}$

TAMs have been the subject of research in cervical cancer. Ding et al found that an increase in macrophages in the tumor stroma was significantly associated with lymphatic metastasis. $^{141}$ The mRNA expression levels of IL-1 $\beta$ and IL-8 in cervical cancer cells cocultured with macrophages were increased compared with those in cervical cancer cells cultured alone. Moreover, the mRNA expression levels of VEGF-C and VEGF-A were increased in macrophages cocultured with cervical cancer cells compared with macrophages cultured alone. ${ }^{141}$ Taken together, these results suggest that TAMs promote lymphangiogenesis mainly through interactions with surrounding cervical cancer cells. ${ }^{141}$ Chen et al observed that high counts of CD68+ and CD163+ macrophages were associated with high risk Human Papilloma Virus (hr-HPV) infection and positively correlated with cervical carcinogenesis. A high index of CD163+ macrophages was significantly associated with higher FIGO stages and lymph node metastasis, but there no such association was found for CD68+ macrophages. ${ }^{142}$ This study supported a critical role of TAMs as prospective predictors of hr-HPVrelated cervical carcinogenesis. CD163, as a promising TAM marker, is superior to CD68 for predicting the malignant transformation and metastatic potential of cervical cancer. ${ }^{142}$

\section{Vulvar and Vaginal Cancers}

Vulvar and vaginal cancers are rare gynecological malignancies. Data on SIR in these tumors are limited. ${ }^{143}$ Based on the data of 64 females with vulvar squamous cancer, Etras et al found elevated NLR and PLR in the lymph node-positive group. The best cutoff values for predicting lymph node metastasis were 2.81 for NLR (84.5\% sensitivity and $89.5 \%$ specificity) and 139.5 for PLR $(68.9 \%$ sensitivity and $89.5 \%$ specificity). ${ }^{144}$ Subsequently, Six et al confirmed that females with vulvar cancer with lymph node involvement had elevated serum CRP levels compared with lymph node-negative patients; however, there was no association with OS. ${ }^{145}$

\section{Correlations Between SIR and Well-Established Cancer Markers in Female Genital Organ Malignancies}

CA-125 (cancer antigen 125), also known as mucin 16 or MUC16, is a protein that in humans is encoded by the MUC16 gene. ${ }^{146} \mathrm{CA}-125$ has applications as a tumor marker or biomarker that may be elevated in the blood of 
some patients with specific types of cancers, or in other conditions that are benign. Increased CA-125 levels are found in, for example, endometrial cancer, breast cancer, liver cancer, and pancreatic cancer, as well as in conditions unrelated to tumors, including heart failure and cirrhosis. The CA-125 test is performed mainly in the diagnosis of ovarian cancer. A high concentration of this protein occurs in $80 \%$ of women diagnosed with this type of cancer. ${ }^{147}$ Testing blood levels of CA-125 has been proposed as useful in the treatment and monitoring of ovarian cancer, but the exact prognostic value of CA-125 levels is not entirely clear. A limited number of studies found that CA-125 levels were lower in ovarian clear cell carcinoma (OCCC) than in other histologic types and did not reflect clinical outcomes of patients with OCCC. ${ }^{146}$ Few studies have compared SIR markers with CA-125. Kim et al found that CA-125 levels were best for predicting advancedstage disease, suboptimal debulking, and platinum resistance, and that PLR and NLR may be the most effective predictors of non-complete response and PFS in patients with OCCC. Although most leukocyte differential counts and SIR markers were statistically significant in predicting advanced-stage disease, suboptimal debulking, and platinum resistance, CA-125 levels had the highest sensitivity, specificity, positive and negative prognostic value, and accuracy. ${ }^{146}$ Specific leukocyte differential counts (neutrophils, monocytes, and platelets), and SIR markers (NLR, MLR, and PLR) increased proportionally with a growing burden of inflammation such as that in cancer, whereas CA-125 levels were relatively low in the early stages of ovarian cancer in comparison with other histologic types of epithelial ovarian cancer because of the apparent initial smaller volume of disease, as well as fundamental differences in the biology of malignancy. ${ }^{146,147}$

Carcinoembryonic antigen (CEA) is also a recognized predictive marker in many cancers. CEA is mainly used as a tumor marker to monitor treatment of colorectal carcinoma, to identify recurrence after surgical resection, for

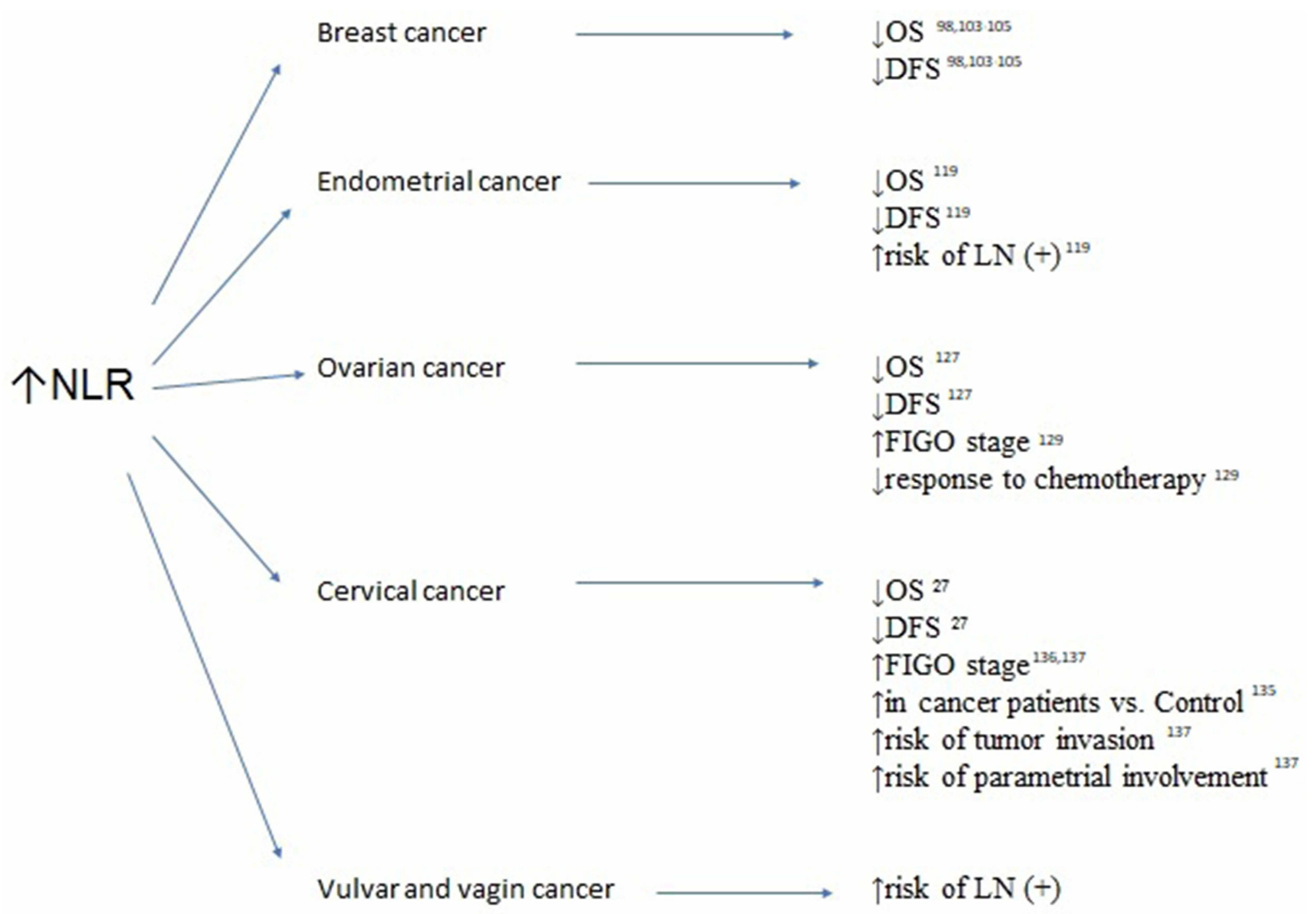

Figure I Association between elevated neutrophil-to-lymphocyte ratio (NRL), survivals and clinicopathological features in breast, endometrial, ovarian, cervical, vulvar and vaginal cancers.

Abbreviations: OS, overall survival; DFS, disease-free survival; LN(+), lymph node involvement; FIGO, International Federation of Gynecologists and Obstetricians. 


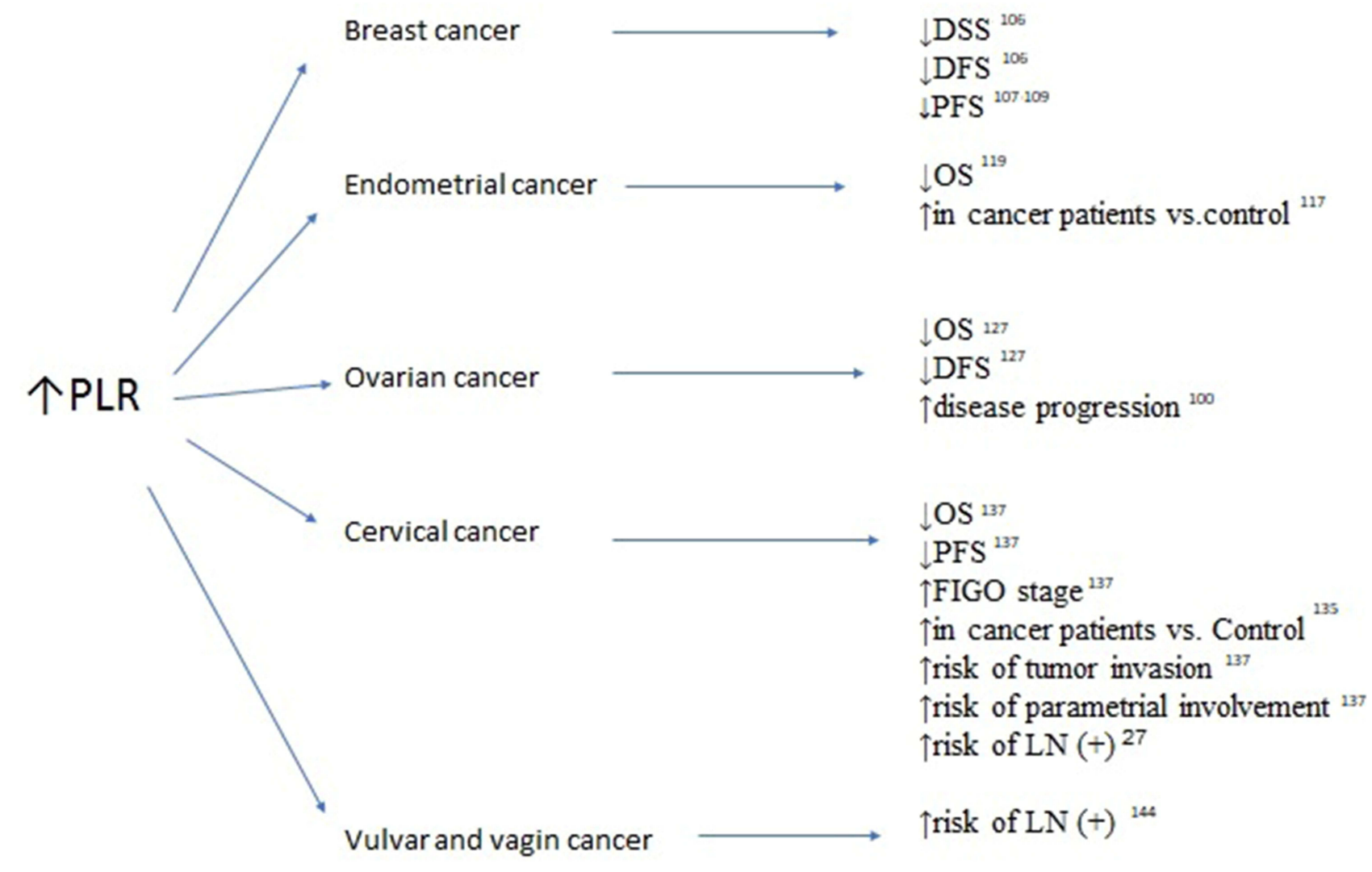

Figure 2 Association between elevated platelet-to-lymphocyte ratio (PRL), survivals and clinicopathological features in breast, endometrial, ovarian, cervical, vulvar and vaginal cancers.

Abbreviations: OS, overall survival; DFS, disease-free survival; PFS, progression-free survival; LN(+), lymph node involvement; FIGO, International Federation of Gynecologists and Obstetricians.

staging, or to localize cancer spread through measurement of biological fluids. ${ }^{148}$ CEA levels may also be raised in breast carcinoma. Studies comparing SIR markers with CEA found that levels of inflammatory markers were significantly associated with CEA levels. In colorectal cancer, prechemotherapy levels of systemic inflammatory markers and CEA were associated with OS and/or PFS. ${ }^{148}$ High prechemotherapy NLR, mGPS, and CEA levels independently predicted poor survival and chemotherapy response. CEA response was also an independent prognostic marker for OS and PFS. High NLR and mGPS were correlated with elevated CEA levels. Therefore the patterns of changes in NLR and CEA levels can be used as prognostic and predictive markers for chemotherapy response. ${ }^{148}$

CA 15-3 (carcinoma antigen 15-3) is a tumor marker for many types of cancer, most notably breast cancer. This antigen is present in normal breast epithelial cells and in breast cancer cells. ${ }^{149}$ The use of CA 15-3 in the detection of breast cancer is limited. Increases in its concentration are observed depending on the stage of the disease in $30-40 \%$ of non- metastatic breast cancer cases and $70 \%$ of metastatic breast cancer cases. ${ }^{149}$ This marker is not suitable for screening for breast cancer; however, it is useful, especially in combination with CEA, in monitoring the effectiveness of treatment and diagnosing relapse. ${ }^{150}$ Only a few studies have compared CA 15-3 with markers of systemic inflammatory response. A study comparing CA 15-3 with the inflammation marker sFas (circulating soluble Fas) found that sFas was more useful for monitoring the response of breast cancer patients to surgery and chemotherapy if the effects of systemic inflammatory reactions were excluded. ${ }^{151}$

\section{The Role of Chronic Inflammatory Reaction in Neoplastic Disease Relapse}

The inflammatory response may play a part in tumor formation and disease relapse after therapy. Surgical intervention may create a favorable microenvironment for local recurrence and accelerate the process of distant 


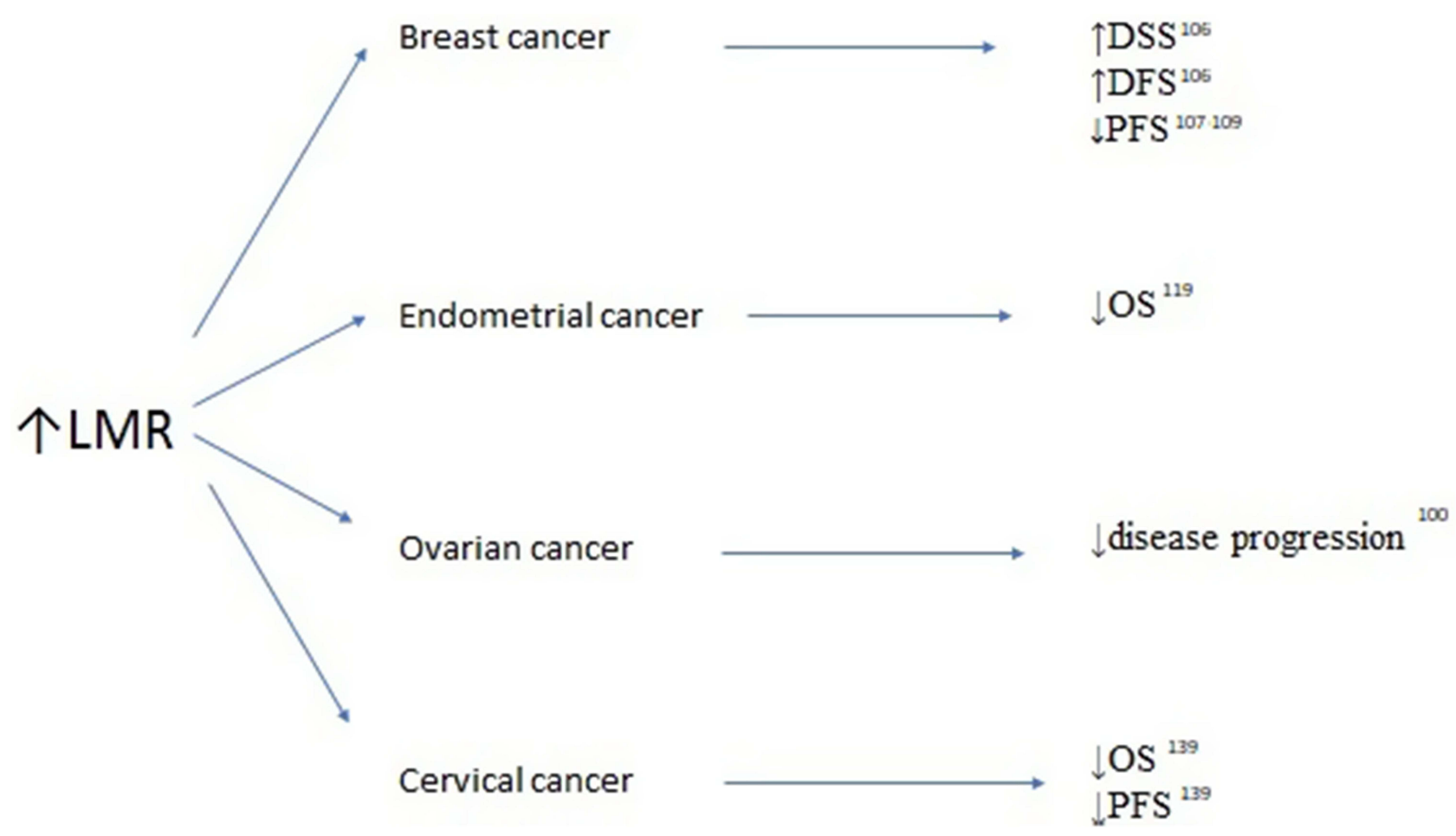

Figure 3 Association between elevated lymphocyte-to-monocyte ratio (LMR) survivals and clinicopathological features in breast, endometrial, ovarian and cervical cancers. Abbreviations: OS, overall survival; DFS, disease-free survival; PFS, progression-free survival.

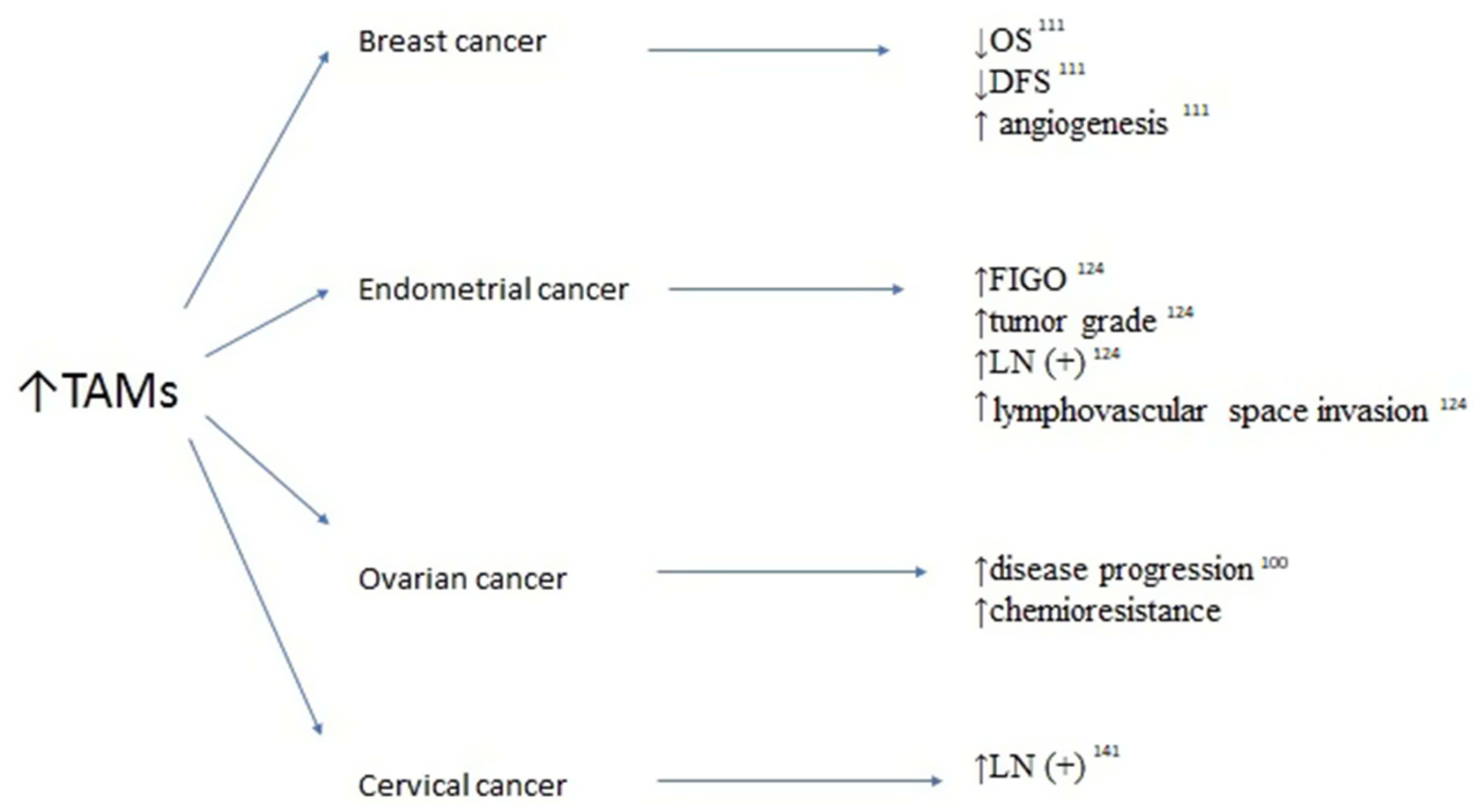

Figure 4 Association between elevated tumor-associated macrophages (TAMS) survivals and clinicopathological features in breast, endometrial, ovarian and cervical cancers.

Abbreviations: OS, overall survival; DFS, disease-free urvival; LN(+), lymph node involvement; FIGO, International Federation of Gynecologists and Obstetricians. 
metastasis. ${ }^{102}$ Clinical data obtained from patients who underwent delayed surgical resection of breast cancer supports the notion that surgery and the resulting healing response may induce the development of distant metastases. ${ }^{103,104}$ The inflammatory response has a very important role in this process. In a mouse model, it was shown that tumor recurrence was associated with a local inflammatory response and SIR characterized by excessive production of pro-inflammatory cytokines (eg, IL1B, IL6, and IL8) and the mobilization of myeloid cells into the circulation. ${ }^{104}$ Monocytes, which differentiate into intratumor macrophages (particularly TAMs), secreting factors that stimulate tumor regrowth and angiogenetic factors, have a special role in this process. ${ }^{106}$

Therefore, the overproduction of pro-inflammatory cytokines is associated with the risk of relapse and further disease progression by inducing resistance to chemotherapy. Pro-inflammatory cytokines (eg, IL1 $\beta$, IL6, and IL8) may be predictive markers of the incidence and stage of cancer, as well as prognostic markers of DFS and OS. ${ }^{115}$

Research on the use of anti-inflammatory drugs after surgery has been initiated to investigate the SIR-to-healing process following tumor resection. It has been shown that the use of such agents can improve treatment outcomes in patients with cancer and reduce the risk of disease recurrence after breast cancer surgery. ${ }^{107}$ These findings are consistent with the results of a retrospective analysis, which showed that the perioperative administration of antiinflammatory analgesics reduced the frequency of metastasis and early relapse in patients with breast cancer. ${ }^{105}$

\section{Conclusions}

SIR is an unfavorable prognostic factor in numerous malignant neoplasms. It contributes to the emergence and progression of disease and is also involved in the occurrence of relapse after anti-cancer treatment (Figures 1-4). Assessment of the inflammatory process can be performed using a number of indicators. As risk stratification is essential in the clinical management of neoplastic disease, the evaluation of the factors described in this article may be promising for the clinical management of patients with neoplastic disease and the development of new therapeutic targets for anti-cancer therapy.

\section{Abbreviations}

CA-125, carcinoma antigen 125; CA 15-3, carcinoma antigen 15-3; CAR, C-reactive protein-to-albumin ratio; CEA, carcinoembryonic antigen; CRP, $\mathrm{C}$ reactive protein; DFS, diseasefree survival; MNR, monocyte-to-lymphocyte ratio; NLR, neutrophil-to-lymphocyte ratio; OS, overall survival; PFS, progression-free survival; PLR, platelet-to-lymphocyte ratio; SII, systemic inflammatory index; SIR, systemic inflammatory reaction; TAMs, tumor-associated macrophages.

\section{Author Contributions}

All authors made substantial contributions to conception and design, acquisition of data, or analysis and interpretation of data; took part in drafting the article or revising it critically for important intellectual content; agreed to submit to the current journal; gave final approval of the version to be published; and agree to be accountable for all aspects of the work.

\section{Funding}

There is no funding to report.

\section{Disclosure}

The authors report no conflicts of interest in this work.

\section{References}

1. Dunn GP, Bruce AT, Ikeda H, Old LJ, Schreiber RD. Cancer immunoediting: from immunosurveillance to tumor escape. Nat Immunol. 2002;3:991-998. doi:10.1038/ni1102-991

2. Dunn GP, Old LJ, Schreiber RD. The immunobiology of cancer immunosurveillance and immunoediting. Immunity. 2004;21:137-148. doi:10.1016/j.immuni.2004.07.017

3. Zou W. Immunosupressive networks in the tumor environment and their therapeutic relevance. Nat Rev Cancer. 2005;5:247-263. doi:10.1038/nrc1586

4. Kim R, Emi M, Tanabe K, Arihiro K. Tumor-driven evolution of immunosuppressive networks during malignant progression. Cancer Res. 2006;66:5527-5536. doi:10.1158/0008-5472.CAN$05-4128$

5. Balkwill F, Mantovani A. Inflammation and cancer: back to Virchow? Lancet. 2001;357(9255):539-545. doi:10.1016/S01406736(00)04046-0

6. Coussens LM, Werb Z. Inflammation and cancer. Nature. 2002;420:860-867. doi:10.1038/nature01322

7. Balkwill F, Charles KA, Mantovani A. Smoldering and polarized inflammation in the initiation and promotion of malignant disease. Cancer Cell. 2005;7:211-217. doi:10.1016/j.ccr.2005.02.013

8. Grivennikov SI, Greten FR, Karin M. Immunity, inflammation, and cancer. Cell. 2010;140(6):883-899. doi:10.1016/j. cell.2010.01.025

9. Aksoy E, Karakurt Z, Gungor S, et al. Neutrophil to lymphocyte ratio is a better indicator of COPD exacerbation severity in neutrophilic endotypes than eosinophilic endotypes. Int J Chron Obstruct Pulmon Dis. 2018;13:2721-2730. doi:10.2147/COPD. S170353

10. Espinosa E, Feliu J, Zamora P, et al. Serum albumin and other prognostic factors related to response and survival in patients with advanced non-small cell lung cancer. Lung Cancer. 1995;12(12):67-76. doi:10.1016/0169-5002(95)00407-R

11. Jones JM, McGonigle NC, McAnespie M, Cran GW, Graham AN. Plasma fibrinogen and serum C-reactive protein are associated with non-small cell lung cancer. Lung Cancer. 2006;53(1):97-101. doi:10.1016/j.lungcan.2006.03.012 
12. Heys SD, Walker LG, Deehan DJ, Eremin OE. Serum albumin: a prognostic indicator in patients with colorectal cancer. $J R$ Coll Surg Edinb. 1998;43(3):163-168.

13. Lai CC, You JF, Yeh CY, et al. Low preoperative serum albumin in colon cancer: a risk factor for poor outcome. Int $J$ Colorectal Dis. 2011;26(4):473-481. doi:10.1007/s00384-010-1113-4

14. Dutta S, Crumley ABC, Fullarton GM, Horgan PG, McMillan DC. Comparison of the prognostic value of tumour and patient related factors in patients undergoing potentially curative resection of gastric cancer. Am J Surg. 2012;204 (3):294-299. doi:10.1016/j.amjsurg.2011.10.015

15. Jiang $X$, Hiki N, Nunobe $S$, et al. Prognostic importance of the inflammation-based Glasgow prognostic score in patients with gastric cancer. Br J Cancer. 2012;107(2):275-279. doi:10.1038/bjc.2012.262

16. Hashimoto K, Ikeda Y, Korenaga D, et al. The impact of preoperative serum C-reactive protein on the prognosis of patients with hepatocellular carcinoma. Cancer. 2005;103(9):1856-1864. doi:10.1002/cncr.20976

17. Kinoshita A, Onoda H, Imai N, et al. Comparison of the prognostic value of inflammation-based prognostic scores in patients with hepatocellular carcinoma. $B r \quad J$ Cancer. 2012;107 (6):988-993. doi:10.1038/bjc.2012.354

18. Crumley ABC, McMillan DC, McKernan M, Going JJ, Shearer CJ, Stuart RC. An elevated C-reactive protein concentration, prior to surgery, predicts poor cancer-specific survival in patients undergoing resection for gastro-oesophageal cancer. $\mathrm{Br}$ $J$ Cancer. 2006;94(11):1568-1571. doi:10.1038/sj.bjc.6603150

19. Crumley ABC, McMillan DC, McKernan M, McDonald AC, Stuart RC. Evaluation of an inflammation-based prognostic score in patients with inoperable gastro-oesophageal cancer. $\mathrm{Br}$ J Cancer. 2006;94(5):637-641. doi:10.1038/sj.bjc.6602998

20. Lis CG, Grutsch JF, Vashi PG, Lammersfeld CA. Is serum albumin an independent predictor of survival in patients with breast cancer? JPEN J Parenter Enteral Nutr. 2003;27(1):10-15. doi:10.1177/014860710302700110

21. Al Murri AM, Bartlett JMS, Canney PA, Doughty JC, Wilson C, McMillan DC. Evaluation of an inflammation based prognostic score (GPS) in patients with metastatic breast cancer. $\mathrm{Br}$ J Cancer. 2006;94(2):227-230. doi:10.1038/sj.bjc.6602922

22. Azab B, Bhatt VR, Phookan J, et al. Usefulness of the neutrophil-to-lymphocyte ratio in predicting short- and long-term mortality in breast cancer patients. Ann Surg Oncol. 2012;19(1):217-224. doi:10.1245/s10434-011-1814-0

23. Parker D, Bradley C, Bogle SM, et al. Serum albumin and CA125 are powerful predictors of survival in epithelial ovarian cancer. $B J O G$. 1994;101(10):888-893. doi:10.1111/j.1471-0528.1994.tb13550.x

24. Hefler LA, Concin N, Hofstetter G, et al. Serum C-reactive protein as independent prognostic variable in patients with ovarian cancer. Clin Cancer Res. 2008;14(3):710-714. doi:10.1158/ 1078-0432.CCR-07-1044

25. Cho H, Hur HW, Kim SW, et al. Pre-treatment neutrophil to lymphocyte ratio is elevated in epithelial ovarian cancer and predicts survival after treatment. Cancer Immunol Immunother. 2009;58(1):15-23. doi:10.1007/s00262-008-0516-3

26. Polterauer S, Grimm C, Seebacher V, et al. The inflammation-based Glasgow prognostic score predicts survival in patients with cervical cancer. Int J Gynecol Cancer. 2010;20 (6):1052-1057. doi:10.1111/IGC.0b013e3181e64bb1

27. Lee YY, Choi CH, Kim HJ, et al. Pretreatment neutrophil: lymphocyteratio as a prognostic factor in cervical carcinoma. Anticancer Res. 2012;32(4):1555-1561.

28. La Torre M, Nigri G, Cavallini M, Mercantini P, Ziparo V, Ramacciato G. The Glasgow prognostic score as a predictor of survival in patients with potentially resectable pancreatic adenocarcinoma. Ann Surg Oncol. 2012;19(9):2917-2923. doi:10.1245/s10434-012-2348-9
29. Lehrer S, Diamond EJ, Mamkine B, Droller MJ, Stone NN, Stock RG. C-reactive protein is significantly associated with prostate-specific antigen and metastatic disease in prostate cancer. BJU Int. 2005;95(7):961-962. doi:10.1111/j.1464410X.2005.05447.x

30. Karakiewicz PI, Hutterer GC, Trinh QD, et al. C-reactive protein is an informative predictor of renal cell carcinoma specific mortality: a European study of 313 patients. Cancer. 2007;110 (6):1241-1247. doi:10.1002/cncr.22896

31. Hilmy M, Bartlett JMS, Underwood MA, McMillan DC. The relationship between the systemic inflammatory response and survival in patients with transitional cell carcinoma of the urinary bladder. $B r \quad J$ Cancer. 2005;92(4):625-627. doi:10.1038/sj. bjc. 6602406

32. Gasparyan AY, Ayvazyan L, Mukanova U, Yessirkepov M, Kitas GD. The platelet-to-lymphocyte ratio as an inflammatory marker in rheumatic diseases. Ann Lab Med. 2019;39 (4):345-357. doi:10.3343/alm.2019.39.4.345

33. Jin Z, Cai G, Zhang P, et al. The value of the neutrophil-tolymphocyte ratio and platelet-to-lymphocyte ratio as complementary diagnostic tools in the diagnosis of rheumatoid arthritis: a multicenter retrospective study. J Clin Lab Anal. 2021;35(1): e23569. doi:10.1002/jcla.23569

34. Chandrashekara S, Lingaraju DC, Renuka P, Anupama KR. Potential of neutrophil to lymphocyte ratio in predicting sustained remission in rheumatoid arthritis compared to other immune activation markers. Indian $J$ Med Res. 2020;152(3):234-243. doi:10.4103/ijmr.IJMR_1676_18

35. Gökmen F, Akbal A, Reşorlu H, et al. Neutrophil-lymphocyte ratio connected to treatment options and inflammation markers of ankylosing spondylitis. J Clin Lab Anal. 2015;29(4):294-298. doi: $10.1002 /$ jcla. 21768

36. Xu S, Ma Y, Wu M, et al. Neutrophil lymphocyte ratio in patients with ankylosing spondylitis: a systematic review and meta-analysis. Mod Rheumatol. 2020;30(1):141-148. doi:10.1080/14397595.2018.1564165

37. Feng JR, Qiu X, Wang F, et al. Diagnostic value of neutrophil-tolymphocyte ratio and platelet-to-lymphocyte ratio in Crohn's disease. Gastroenterol Res Pract. 2017;2017:3526460. doi:10.1155/ 2017/3526460

38. Mertoglu C, Gunay M. Neutrophil-Lymphocyte ratio and Platelet-Lymphocyte ratio as useful predictive markers of prediabetes and diabetes mellitus. Diabetes Metab Syndr. 2017;Suppl 1: S127-S131. doi:10.1016/j.dsx.2016.12.021

39. Bilge M, Yesilova A, Adas M, Helvaci A. Neutrophil- and platelet- to lymphocyte ratio in patients with Euthyroid Hashimoto's thyroiditis. Exp Clin Endocrinol Diabetes. 2019;127(8):545-549. doi:10.1055/a-0723-3441

40. Tatum D, Taghavi S, Houghton A, Stover J, Toraih E, Duchesne J. Neutrophil-to-lymphocyte ratio and outcomes in Louisiana COVID-19 patients. Shock. 2020;54(5):652-658. doi:10.1097/SHK.0000000000001585

41. Howard R, Scheiner A, Kanetsky PA, Egan KM. Sociodemographic and lifestyle factors associated with the neutrophil-to-lymphocyte ratio. Ann Epidemiol. 2019;38:11-21 e6. doi:10.1016/j.annepidem.2019.07.015

42. Nowak-Markwitz E, Spaczyński M. Rak jajnik - nowe spojrzenie na pochodzenie i histogenezę [Ovarian cancer - modern approach to its origin and histogenesis]. Ginekol Pol. 2012;83:454-457. Polish.

43. Shih L, Kurman RJ. Ovarian tumorigenesis: a proposed model based on morphological and molecular genetic analysis. Am J Pathol. 2004;164:1511-1518. doi:10.1016/S0002-9440(10)63708-X

44. Pollard JW. Tumor - educated macrophages promote tumor progression and metastasis. Nat Rev Cancer. 2004;4:71-78. doi: $10.1038 / \mathrm{nrc1} 256$ 
45. Hofmeister V, Vetter C, Schrama D, Bröcker EB, Becker JC. Tumor stroma-associated antigens for anti-cancer immunotherapy. Cancer Immunol Immunother. 2006;55:481-494. doi:10.1007/s00262-005-0070-1

46. Murdoch C, Giannoudis A, Lewis CE. Mechanisms regulating the recruitment of macrophages into hypoxic areas of tumors and other ischemic tissues. Blood. 2004;104:2224-2234. doi:10.1182/blood-2004-03-1109

47. Mantovani A, Sozzani S, Locati M, Allavena P, Sica A. Macrophage polarization: tumor-associated macrophages as a paradigm for polarized M2 mononuclear phagocytes. Trends Immunol. 2002;23:549-555. doi:10.1016/S1471-4906(02) 02302-5

48. Komohara Y, Fujiwara Y, Ohnishi K, Takeya M. Tumorassociated macrophages: potential therapeutic targets for anti-cancer therapy. Adv Drug Deliv Rev. 2016;99(Pt B):180-185. doi:10.1016/j.addr.2015.11.009

49. Condeelis J, Pollard JW. Macrophages: obligate partners for tumor cell migration, invasion, and metastasis. Cell. 2006;124:263-266. doi:10.1016/j.cell.2006.01.007

50. Lewis CE, Pollard JW. Distinct role of macrophages in different tumor microenvironments. Cancer Res. 2006;66:605-612. doi:10.1158/0008-5472.CAN-05-4005

51. Kim J, Bae JS. Tumor-associated macrophages and neutrophil in tumor microenvironment. Mediators Inflamm. 2016;2016:6058147. doi:10.1155/2016/6058147

52. Mantovani A, Allavena P, Sica A, Balkwill F. Cancer-related inflammation. Nature. 2008;454(7203):436-444. doi:10.1038/ nature 07205

53. Kelly MG, Alvero AB, Chen R, et al. TLR-4 signaling promotes tumor growth and paclitaxel chemoresistance in ovarian cancer Cancer Res. 2006;66(7):3859-3868. doi:10.1158/0008-5472. CAN-05-3948

54. Reuter S, Gupta SC, Chaturvedi MM, Aggarwal BB. Oxidative stress, inflammation, and cancer: how are they linked? Free Radic Biol Med. 2010;49(11):1603-1616.

55. de Sá Junior PL, Câmara DAD, Porcacchia AS, et al. The roles of ROS in cancer heterogeneity and therapy. Oxid Med Cell Longev. 2017;2017:1-12. doi:10.1155/2017/2467940

56. Galdiero MR, Bonavita E, Barajon I, et al. Tumor associated macrophages and neutrophils in cancer. Immunobiology. 2013;218(11):1402-1410. doi:10.1016/j.imbio.2013.06.003

57. Mantovani A. Macrophages, neutrophils, and cancer: a double edged sword. New J Sci. 2014;2014:1-14. doi:10.1155/2014/ 271940

58. Fridlender ZG, Albelda SM. Tumor-associated neutrophils: friend or foe? Carcinogenesis. 2012;33(5):949-955. doi:10.1093/carcin/ bgs 123

59. Sagiv JY, Michaeli J, Assi S, et al. Phenotypic diversity and plasticity in circulating neutrophil subpopulations in cancer. Cell Rep. 2015;10:562-573. doi:10.1016/j.celrep.2014.12.039

60. Gabay C, Kushner I. Acute-phase proteins and other systemic responses to inflammation. $N$ Engl J Med. 1999;340(6):448-454. doi:10.1056/NEJM199902113400607

61. Kim ES, Kim SY, Koh M, et al. C-reactive protein binds to integrin $\alpha 2$ and $\mathrm{Fc} \gamma$ receptor I, leading to breast cell adhesion and breast cancer progression. Oncogene. 2018;37(1):28-38. doi:10.1038/onc. 2017.298

62. Kim ES, Cha $\mathrm{Y}$, Ham $\mathrm{M}$, et al. Inflammatory lipid sphingosine-1-phosphate upregulates $\mathrm{C}$-reactive protein via $\mathrm{C} /$ EBPbeta and potentiates breast cancer progression. Oncogene. 2014;33(27):3583-3593. doi:10.1038/onc.2013.319

63. Allin KH, Nordestgaard BG, Flyger H, Bojesen SE. Elevated pre-treatment levels of plasma C-reactive protein are associated with poor prognosis after breast cancer: a cohort study. Breast Cancer Res. 2011;13(3):R55. doi:10.1186/bcr2891
64. Mantzorou M, Koutelidakis A, Theocharis S, Giaginis C. Clinical value of nutritional status in cancer: what is its impact and how it affects disease progression and prognosis? Nutr Cancer. 2017;69 (8):1151-1176. doi:10.1080/01635581.2017.1367947

65. Gupta D, Lis CG. Pretreatment serum albumin as a predictor of cancer survival: a systematic review of the epidemiological literature. Nutr J. 2010;9:69. doi:10.1186/1475-2891-9-69

66. Rahman MM, Ahsan MA, Monalisa NN, Rahman K. Influence of socioeconomic status and BMI on the quality of life after mastectomy in Bangladeshi breast cancer patients in a public hospital. Jpn J Clin Oncol. 2014;44(12):1150-1157. doi:10.1093/jjco/ hyu144

67. Roxburgh CS, McMillan DC. Role of systemic infammatory response in predicting survival in patients with primary operable cancer. Future Oncol. 2010;6(1):149-163. doi:10.2217/ fon. 09.136

68. Viganò A, Bruera E, Jhangri GS, Newman SC, Fields AL, Suarez-Almazor ME. Clinical survival predictors in patients with advanced cancer. Arch Intern Med. 2000;160(6):861-868.

69. Sica A, Erreni M, Allavena P, Porta C. Macrophage polarization in pathology. Cell Mol Life Sci. 2015;72(21):4111-4126. doi:10.1007/s00018-015-1995-y

70. Paesmans M, Sculier JP, Libert P, et al. Prognostic factors for survival in advanced non-small-cell lung cancer: univariate and multivariate analyses including recursive partitioning and amalgamation algorithms in 1052 patients. The European Lung Cancer Working Party. J Clin Oncol. 1995;13(5):1221-1230. doi:10.1200/JCO.1995.13.5.1221

71. Teramukai S, Kinato T, Kishida Y, et al. Pretreatment neutrophil count as an independent prognostic factor in advanced non-smallcell lung cancer: an analysis of Japan multinational trial organization LC00-03. Eur J Cancer. 2009;45:1950-1958. doi:10.1016/j. ejca.2009.01.023

72. Lu CC, Chang KW, Chou FC, et al. Association of pretreatment thrombocytosis with disease progression and survival in oral squamous cell carcinoma. Oral Oncol. 2007;43:282-288. doi:10.1016/j.oraloncology.2006.03.010

73. Pedersen LM, Milman N. Diagnostic significance of platelet count and other blood analyses in patients with lung cancer. Oncol Rep. 2003;10:213-216.

74. Unal D, Eroglu C, Kurtul N, et al. Are neutrophil/lymphocyte and platelet/lymphocyte rates in patients with non-small cell lung cancer associated with treatment response and prognosis. Asian Pac J Cancer Prev. 2013;14:5237-5242. doi:10.7314/ APJCP.2013.14.9.5237

75. Sato H, Tsubosa Y, Kawano T. Correlation between the pretherapeutic neutrophil to lymphocyte ratio and pathologic response to neoadjuvant chemotherapy in patients with advanced esophageal cancer. World J Surg. 2012;36(3):617-622. doi:10.1007/s00268011-1411-1

76. Williams KA, Labidi-Galy I, Terry KL, et al. Prognostic significance and predictors of the neutrophil-to-lymphocyte ratio in ovarian cancer. Gynecol Oncol. 2014;132(3):542-550. doi:10.1016/j.ygyno.2014.01.026

77. Zhou Q, Hong L, Zuo MZ, He Z. Prognostic significance of neutrophil to lymphocyte ratio in ovarian cancer: evidence from 4910 patients. Oncotarget. 2017;8(40):68938-68949. doi:10.18632/oncotarget.20196

78. Gu L, Li H, Chen L, et al. Prognostic role of lymphocyte to monocyte ratio for patients with cancer: evidence from a systematic review and meta-analysis. Oncotarget. 2016;7 (22):31926-31942. doi:10.18632/oncotarget.7876

79. Chen JH, Zhai ET, Yuan YJ, et al. Systemic immune-inflammation index for predicting prognosis of colorectal cancer. World J Gastroenterol. 2017;23(34):6261-6272. doi:10.3748/wjg.v23.i34.6261 
80. Hu B, Yang XR, Xu Y, et al. Systemic immune-inflammation index predicts prognosis of patients after curative resection for hepatocellular carcinoma. Clin Cancer Res. 2014;20 (23):6212-6222. doi:10.1158/1078-0432.CCR-14-0442

81. Fairclough E, Cairns E, Hamilton J, Kelly C. Evaluation of a modified early warning system for acute medical admissions and comparison with C-reactive protein/albumin ratio as a predictor of patient outcome. Clin Med (Lond). 2009;9 (1):30-33. doi:10.7861/clinmedicine.9-1-30

82. Ranzani OT, Zampieri FG, Forte DN, Azevedo LC, Park M. C-reactive protein/albumin ratio predicts 90-day mortality of septic patients. PLoS One. 2013;8(3):e59321. doi:10.1371/journal. pone. 0059321

83. Kinoshita A, Onoda H, Imai N, et al. The C-reactive protein/ albumin ratio, a novel inflammation-based prognostic score, predicts outcomes in patients with hepatocellular carcinoma. Ann Surg Oncol. 2015;22(3):803-810. doi:10.1245/s10434014-4048-0

84. Liu X, Sun X, Liu J, et al. Preoperative C-reactive protein/albumin ratio predicts prognosis of patients after curative resection for gastric cancer. Transl Oncol. 2015;8(4):339-345. doi:10.1016/j. tranon.2015.06.006

85. Xu XL, Yu HQ, Hu W, Song Q, Mao WM. A novel inflammation-based prognostic score, the C-reactive protein/albumin ratio predicts the prognosis of patients with operable esophageal squamous cell carcinoma. PLoS One. 2015;10(9):e0138657. doi:10.1371/journal.pone. 0138657

86. Liu $\mathrm{Y}$, Chen $\mathrm{S}$, Zheng $\mathrm{C}$, et al. The prognostic value of the preoperative c-reactive protein/albumin ratio in ovarian cancer. BMC Cancer. 2017;17(1):285. doi:10.1186/s12885-017-3220-x

87. Zhou L, Ma S, Balde AI, et al. A retrospective propensity score matched study of the preoperative C-reactive protein to albumin ratio and prognosis in patients with resectable non-metastatic breast cancer. Med Sci Monit. 2019;25:4342-4352. doi:10.12659/MSM.913684

88. Onitilo AA, Engel JM, Stankowski RV, et al. High-sensitivity C-reactive protein (hs-CRP) as a biomarker for trastuzumab-induced cardiotoxicity in HER2- positive early-stage breast cancer: a pilot study. Breast Cancer Res Treat. 2012;134(1):291-298. doi:10.1007/s10549-012-2039-z

89. Forrest LM, McMillian DC, McArdle CS et al. Evaluation of cumulatrive prognostic scores based on the systemic inflammatory response in patients with inoperable non-small-cell lung cancer. Br J Cancer. 2003; 89:1028-1030

90. Dolan RD, Lim J, McSorley ST, et al. The role of the systemic inflammatory response in predicting outcomes in patients with operable cancer: systematic review and meta-analysis. Sci Rep. 2017;7(1):16717.

91. Staton CA, Brown NJ, Lewis CE. The role of fibrinogen and related fragments in tumour angiogenesis and metastasis. Expert Opin Biol Ther. 2003;3(7):1105-1120. doi:10.1517/ 14712598.3.7.1105

92. Martino MM, Briquez PS, Ranga A, et al. Heparin-binding domain of fibrin(ogen) binds growth factors and promotes tissue repair when incorporated within a synthetic matrix. Proc Natl Acad Sci U S A. 2013;110(12):4563-4568. doi:10.1073/ pnas. 1221602110

93. Xu Q, Yan Y, Gu S, et al. A novel inflammation-based prognostic score: the fibrinogen/albumin ratio predicts prognoses of patients after curative resection for hepatocellular carcinoma. J Immunol Res. 2018;2018:4925498. doi:10.1155/2018/4925498

94. Perisanidis C, Psyrri A, Cohen EE, et al. Prognostic role of pretreatment plasma fibrinogen in patients with solid tumors: a systematic review and meta-analysis. Cancer Treat Rev. 2015;41(10):960-970. doi:10.1016/j.ctrv.2015.10.002
95. Desgrosellier JS, Cheresh DA. Integrins in cancer: biological implications and therapeutic opportunities. Nat Rev Cancer. 2010;10(1):9-22. doi:10.1038/nrc2748

96. Tan Z, Zhang M, Han Q, et al. A novel blood tool of cancer prognosis in esophageal squamous cell carcinoma: the fibrinogen/ albumin ratio. J Cancer. 2017;8(6):1025-1029. doi:10.7150/ jca. 16491

97. Li X, An B, Zhao Q, et al. Combined fibrinogen and neutrophillymphocyte ratio as a predictive factor in resectable colorectal adenocarcinoma. Cancer Manag Res. 2018;10:6285-6294. doi:10.2147/CMAR.S161094

98. Fahad Ullah M. Breast cancer: current perspectives on the disease status. Adv Exp Med Biol. 2019;1152:51-64.

99. Ethier JL, Desautels D, Templeton A, Shah PS, Amir E. Prognostic role of neutrophil-to-lymphocyte ratio in breast cancer: a systematic review and meta-analysis. Breast Cancer Res. 2017;19(1):2. doi:10.1186/s13058-016-0794-1

100. Koh YW, Lee HJ, Ahn JH, Lee JW, Gong G. Prognostic significance of the ratio of absolute neutrophil to lymphocyte counts for breast cancer patients with ER/PR-positivity and HER2-negativity in neoadjuvant setting. Tumor Biol. 2014;35 (10):9823-9830. doi:10.1007/s13277-014-2282-5

101. Corbeau I, Jacot W, Guiu S. Neutrophil to lymphocyte ratio as prognostic and predictive factor in breast cancer patients: a systematic review. Cancers (Basel). 2020;12(4):958. doi:10.3390/cancers 12040958

102. Cullinane C, Creavin B, O'Leary DP, et al. Can the neutrophil to lymphocyte ratio predict complete pathologic response to neoadjuvant breast cancer treatment? A systematic review and meta-analysis. Clin Breast Cancer. 2020;20(6):e675-e681. doi:10.1016/j.clbc.2020.05.008

103. Iwase $T$, Sangai $T$, Sakakibara $M$, et al. An increased neutrophil-to-lymphocyte ratio predicts poorer survival following recurrence for patients with breast cancer. Mol Clin Oncol. 2017;6 (2):266-270. doi:10.3892/mco.2016.1101

104. Miyagawa Y, Araki K, Bun A, et al. Significant association between low baseline neutrophil-to-lymphocyte ratio and improved progression-free survival of patients with locally advanced or metastatic breast cancer treated with eribulin but not with nab-paclitaxel. Clin Breast Cancer. 2018;18 (5):400-409. doi:10.1016/j.clbc.2018.03.002

105. Takuwa H, Tsuji W, Yamamoto Y, et al. Low neutrophil-lymphocyte ratio correlates with extended survival in patients with metastatic breast cancer who achieved clinically complete response following multidisciplinary therapy: a retrospective study. Oncol Lett. 2018;15(5):6681-6687. doi:10.3892/ol.2018.8145

106. Cho U, Park HS, Im SY, et al. Prognostic value of systemic inflammatory markers and development of a nomogram in breast cancer. PLoS One. 2018;13(7):e0200936. doi:10.1371/journal. pone. 0200936

107. Araki K, Ito Y, Fukada I, et al. Predictive impact of absolute lymphocyte counts for progression-free survival in human epidermal growth factor receptor 2-positive advanced breast cancer treated with pertuzumab and trastuzumab plus eribulin or nab-paclitaxel. BMC Cancer. 2018;18(1):982. doi:10.1186/ s12885-018-4888-2

108. Vernieri C, Mennitto A, Prisciandaro M, et al. The neutrophil-tolymphocyte and platelet-to-lymphocyte ratios predict efficacy of platinum-based chemotherapy in patients with metastatic triple negative breast cancer. Sci Rep. 2018;8(1):1-10. doi:10.1038/ s41598-018-27075-z

109. Ji Y, Wang H. Prognostic prediction of systemic immune-inflammation index for patients with gynecological and breast cancers: a meta-analysis. World J Surg Oncol. 2020;18 (1):197. doi:10.1186/s12957-020-01974-w 
110. Chen L, Kong X, Wang Z, et al. Pretreatment systemic inflammation response index in patients with breast cancer treated with neoadjuvant chemotherapy as a useful prognostic indicator. Cancer Manag Res. 2020;12:1543-1567. doi:10.2147/CMAR. S235519

111. Zhao X, Qu J, Sun Y, et al. Prognostic significance of tumorassociated macrophages in breast cancer: a meta- analysis of the literature. Oncotarget. 2017;8(18):30576-30586. doi:10.18632/ oncotarget. 15736

112. Banas T, Juszczyk G, Pitynski K, et al. Incidence and mortality rates in breast, corpus uteri, and ovarian cancers in Poland (1980 2013): an analysis of population-based data in relation to socioeconomic changes. Onco Targets Ther. 2016;9:5521-5530. doi:10.2147/OTT.S112187

113. Cakmak B, Gulucu S, Aliyev N, et al. Neutrophil-lymphocyte and platelet-lymphocyte ratios in endometrial hyperplasia. Obstet Gynecol Sci. 2015;58(2):157-161. doi:10.5468/ogs.2015.58.2.157

114. Ural ÜM, Şehitoğlu İ, Tekin YB, Şahin FK. Neutrophil-tolymphocyte and platelet-to-lymphocyte ratios in patients with endometrial hyperplasia and endometrial cancer. $J$ Obstet Gynaecol Res. 2015;41(3):445-448. doi:10.1111/jog.12536

115. Selen S, Kilic F, Kimyon Comert G, et al. Can preoperative inflammatory markers differentiate endometrial cancer from complex atypical hyperplasia/endometrial intraepithelial neoplasia? $J$ Obstet Gynaecol Res. 2020;46(7):1148-1156. doi:10.1111/ jog. 14314

116. Acmaz G, Aksoy H, Unal D, et al. Are neutrophil/lymphocyte and platelet/lymphocyte ratios associated with endometrial precancerous and cancerous lesions in patients with abnormal uterine bleeding? Asian Pac J Cancer Prev. 2014;15(4):1689-1692. doi:10.7314/APJCP.2014.15.4.1689

117. Pergialiotis V, Oikonomou M, Damaskou V, et al. Platelet to lymphocyte and neutrophil to lymphocyte ratio as predictive indices of endometrial carcinoma: findings from a retrospective series of patients and meta-analysis. J Gynecol Obstet Hum Reprod. 2018;47(10):511-516. doi:10.1016/j.jogoh.2018.08.016

118. NSSN guidelines [homepage on the internet] Clinical Practice Guidelines in Oncology; 2021. Available from: https://www. nccn.org/professionals/physician_gls/default.aspx. Accessed March 20, 2021.

119. Haruma T, Nakamura K, Nishida T. Pre-treatment neutrophil to lymphocyte ratio is a predictor of prognosis in endometrial cancer. Anticancer Res. 2015;35(1):337-343.

120. Kadan Y, Calvino AS, Katz A, Katz S, Moore RG. Predictors for lymph nodes involvement in low risk endometrial cancer. $J$ Obstet Gynaecol. 2017;37(4):514-518. doi:10.1080/ 01443615.2017.1281895

121. Cong R, Kong F, Ma J, Li Q, Wu Q, Ma X. Combination of preoperative neutrophil-lymphocyte ratio, platelet-lymphocyte ratio and monocyte-lymphocyte ratio: a superior prognostic factor of endometrial cancer. BMC Cancer. 2020;20(1):464. doi:10.1186/s12885-020-06953-8

122. Eo WK, Kwon S, Koh SB. The lymphocyte-monocyte ratio predicts patient survival and aggressiveness of endometrial cancer. $J$ Cancer. 2016;7(5):538-545. doi:10.7150/jca.14206

123. Li J, Lin J, Luo Y, Kuang M, Liu Y. Multivariate analysis of prognostic biomarkers in surgically treated endometrial cancer. PLoS One. 2015;10(6):e0130640. doi:10.1371/journal.pone.0130640

124. Kübler K, Ayub TH, Weber SK, et al. Prognostic significance of tumor-associated macrophages in endometrial adenocarcinoma. Gynecol Oncol. 2014;135(2):176-183. doi:10.1016/j. ygyno.2014.08.028

125. Kelly MG, Francisco AM, Cimic A, et al. Type 2 endometrial cancer is associated with a high density of tumor associated macrophages in the stromal compartment. Reprod Sci. 2015;22 (8):948-953. doi:10.1177/1933719115570912
126. Dai D, Liu L, Huang H, et al. Nomograms to predict the density of tumor-infiltrating lymphocytes in patients with high-grade serous ovarian cancer. Front Oncol. 2021;11:590414. doi:10.3389/ fonc. 2021.590414

127. Zhao Z, Zhao X, Lu J, et al. Prognostic roles of neutrophil to lymphocyte ratio and platelet to lymphocyte ratio in ovarian cancer: a meta-analysis of retrospective studies. Arch Gynecol Obstet. 2018;297(4):849-857. doi:10.1007/s00404-018-4678-8

128. Huang QT, Zhou L, Zeng WJ, et al. Prognostic significance of neutrophil-to-lymphocyte ratio in ovarian cancer: a systematic review and meta-analysis of observational studies. Cell Physiol Biochem. 2017;41(6):2411-2418. doi:10.1159/000475911

129. Zhang H, Huo Q, Huang L, Cheng Y, Liu Y, Bao H. Neutrophil-to -lymphocyte ratio in ovarian cancer patients with low CA125 concentration. Biomed Res Int. 2019;2019:8107906.

130. Wu YY, Qin YY, Qin JQ, Zhang X, Lin FQ. Diagnostic value of derived neutrophil-to-lymphocyte ratio in patients with ovarian cancer. J Clin Lab Anal. 2019;33(4):e22833. doi:10.1002/ jcla. 22833

131. Lu C, Zhou L, Ouyang J, Yang H. Prognostic value of lymphocyte-to-monocyte ratio in ovarian cancer: a meta-analysis. Medicine (Baltimore). 2019;98(24):e1587. doi:10.1097/MD.0000000000015876

132. Zhang M, He Y, Sun X, et al. A high M1/M2 ratio of tumor associated macrophages is associated with extended survival in ovarian cancer patients. J Ovarian Res. 2014;7:19. doi:10.1186/ 1757-2215-7-19

133. Yin M, Shen J, Yu S, et al. Tumor-Associated Macrophages (TAMs): a critical activator in ovarian cancer metastasis. Onco Targets Ther. 2019;21(12):8687-8699. doi:10.2147/OTT.S216355

134. Shrestha AD, Neupane D, Vedsted P, Kallestrup P. Cervical cancer prevalence, incidence and mortality in low and middle income countries: a systematic review. Asian Pac J Cancer Prev. 2018;19(2):319-324. doi:10.22034/APJCP.2018.19.2.319

135. Tas M, Yavuz A, Ak M, Ozcelik B. Neutrophil-to-lymphocyte ratio and platelet-to-lymphocyte ratio in discriminating precancerous pathologies from cervical cancer. $J$ Oncol. 2019;2019:2476082. doi:10.1155/2019/2476082

136. Prabawa IPY, Bhargah A, Liwang F, et al. Pretreatment neutrophil-to-lymphocyte ratio (NLR) and platelet-tolymphocyte ratio (PLR) as a predictive value of hematological markers in cervical cancer. Asian Pac J Cancer Prev. 2019;20 (3):863-868. doi:10.31557/APJCP.2019.20.3.863

137. Zhu M, Feng M, He F, et al. Pretreatment neutrophil-lymphocyte and platelet-lymphocyte ratio predict clinical outcome and prognosis for cervical cancer. Clin Chim Acta. 2018;483:296-302. doi:10.1016/j.cca.2018.05.025

138. Zhu J, Wang H, Gao MJ, et al. Prognostic values of lymphocyte and eosinophil counts in resectable cervical squamous cell carcinoma. Future Oncol. 2019;15(30):3467-3481. doi:10.2217/ fon-2018-0879

139. Trinh H, Dzul SP, Hyder J, et al. Prognostic value of changes in neutrophil-to-lymphocyte ratio (NLR), platelet-to-lymphocyte ratio (PLR) and lymphocyte-to-monocyte ratio (LMR) for patients with cervical cancer undergoing definitive chemoradiotherapy (dCRT). Clin Chim Acta. 2020;510:711-716. doi:10.1016/j.cca.2020.09.008

140. Zhang W, Liu K, Ye B, Liang W, Ren Y. Pretreatment C-reactive protein/albumin ratio is associated with poor survival in patients with stage IB-IIA cervical cancer. Cancer Med. 2018;7 (1):105-113. doi:10.1002/cam4.1270

141. Ding H, Cai J, Mao M, et al. Tumor-associated macrophages induce lymphangiogenesis in cervical cancer via interaction with tumor cells. APMIS. 2014;122(11):1059-1069. doi:10.1111/ apm. 12257 
142. Chen X-J, Han L-F, Wu X-G, et al. Clinical significance of CD163+ and CD68+ tumor-associated macrophages in high-risk HPV-related cervical cancer. $J$ Cancer. 2017;8(18):3868-3875. doi:10.7150/jca.21444

143. Banas T, Pitynski K, Jach R, et al. Primary vulvo-vaginal cancers: trends in incidence and mortality in Poland (1999-2012). Gynecol Obstet Invest. 2015;80(4):240-245. doi:10.1159/000381770

144. Ertas IE, Gungorduk K, Akman L, et al. Can preoperative neutrophil: lymphocyteand platelet: lymphocyteratios be used as predictive markers for lymph node metastasis in squamous cell carcinoma of the vulva? Eur J Obstet Gynecol Reprod Biol. 2013;171(1):138-142. doi:10.1016/j.ejogrb.2013.08.023

145. Six L, Polterauer S, Grimm C, et al. C-reactive protein serum levels are closely associated with lymph node status, but not with prognosis in patients with vulvar cancer. Eur J Obstet Gynecol Reprod Biol. 2008;137(2):217-221. doi:10.1016/j. ejogrb.2007.02.020

146. Kim HS, Choi HY, Lee M, et al. Systemic inflammatory response markers and CA-125 levels in ovarian clear cell carcinoma: a Two Center Cohort Study. Cancer Res Treat. 2016;48(1):250-258. doi:10.4143/crt.2014.324
147. Fader AN, Java J, Krivak TC, et al. The prognostic significance of pre- and post-treatment CA-125 in grade 1 serous ovarian carcinoma: a gynecologic Oncology Group study. Gynecol Oncol. 2014;132:560-565. doi:10.1016/j.ygyno.2013.11.016

148. Kim I-H, Lee JE, Yang JH, et al. Clinical significance of changes in systemic inflammatory markers and carcinoembryonic antigen levels in predicting metastatic colorectal cancer prognosis and chemotherapy response. Asia Pac J Clin Oncol. 2018;14 (3):239-246. doi:10.1111/ajco.12784

149. Donepudi MS, Kondapalli K, Amos SJ, Venkanteshan P. Breast cancer statistics and markers. $J$ Cancer Res Ther. 2014;10 (3):506-11.

150. Chernecky CC, Berger BJ. Laboratory Tests and Diagnostic Procedures. Elsevier Health Sciences; 2007:s. 269-270.

151. Hewala TI, Abd El-Monaim NA, Anwar M, Ebied SA. The clinical significance of serum soluble Fas and p53 protein in breast cancer patients: comparison with serum CA 15-3. Pathol Oncol Res. 2012;18(4):841-848. doi:10.1007/s12253-012-9512-1

\section{Publish your work in this journal}

Cancer Management and Research is an international, peer-reviewed open access journal focusing on cancer research and the optimal use of preventative and integrated treatment interventions to achieve improved outcomes, enhanced survival and quality of life for the cancer patient.
The manuscript management system is completely online and includes a very quick and fair peer-review system, which is all easy to use. Visit http://www.dovepress.com/testimonials.php to read real quotes from published authors. 